\title{
AN INVESTIGATION OF READING AND BIMANUAL TAPPING ABILITY IN ADULTS
}

\author{
A thesis submitted to \\ the Faculty of Graduate Studies and Research \\ in Partial Fulfillment of the requirements for the degree \\ Masters of Arts \\ by
}

Jessica Gunnell

Department of Psychology

Carleton University

September 2008

(C)2008 Jessica Gunnell 


$\begin{array}{ll}\begin{array}{l}\text { Library and } \\ \text { Archives Canada }\end{array} & \begin{array}{l}\text { Bibliothèque et } \\ \text { Archives Canada }\end{array} \\ \begin{array}{l}\text { Published Heritage } \\ \text { Branch }\end{array} & \begin{array}{l}\text { Direction du } \\ \text { Patrimoine de l'édition }\end{array} \\ \begin{array}{l}\text { 395 Wellington Street } \\ \text { Ottawa ON K1A 0N4 } \\ \text { Canada }\end{array} & \begin{array}{l}\text { O95, rue Wellington } \\ \text { Ottawa ON K1A 0N4 } \\ \text { Canada }\end{array}\end{array}$

Your file Votre référence ISBN: 978-0-494-43460-4

Our file Notre référence

ISBN: 978-0-494-43460-4

NOTICE:

The author has granted a nonexclusive license allowing Library and Archives Canada to reproduce, publish, archive, preserve, conserve, communicate to the public by telecommunication or on the Internet, loan, distribute and sell theses worldwide, for commercial or noncommercial purposes, in microform, paper, electronic and/or any other formats.

The author retains copyright ownership and moral rights in this thesis. Neither the thesis nor substantial extracts from it may be printed or otherwise reproduced without the author's permission.
AVIS:

L'auteur a accordé une licence non exclusive permettant à la Bibliothèque et Archives Canada de reproduire, publier, archiver, sauvegarder, conserver, transmettre au public par télécommunication ou par l'Internet, prêter, distribuer et vendre des thèses partout dans le monde, à des fins commerciales ou autres, sur support microforme, papier, électronique et/ou autres formats.

L'auteur conserve la propriété du droit d'auteur et des droits moraux qui protège cette thèse. $\mathrm{Ni}$ la thèse ni des extraits substantiels de celle-ci ne doivent être imprimés ou autrement reproduits sans son autorisation.
In compliance with the Canadian

Privacy Act some supporting forms may have been removed from this thesis.

While these forms may be included in the document page count, their removal does not represent any loss of content from the thesis.
Conformément à la loi canadienne sur la protection de la vie privée, quelques formulaires secondaires ont été enlevés de cette thèse.

Bien que ces formulaires aient inclus dans la pagination, il n'y aura aucun contenu manquant.

\section{Canada}




\begin{abstract}
This study re-examined the proposed relationship between temporal processing (as measured by bimanual rhythmic finger tapping) and reading ability in 95 adults. Participants were administered the Bimanual Tapping Task (BTT), measures of reading (Word Identification, Word Attack, Passage Comprehension, Reading Fluency, and Spelling), IQ, phonological processing, rapid naming, and working memory. The BTT was first used to examine the effect of speed and ratio on three groups (Control $n=59$; Dyslexic (DD) $n=25$; Learning Disability and or Attention Deficit Hyperactivity Disorder $(\mathrm{LD} / \mathrm{ADHD}) n=11)$. The results indicated that the tapping variability at a $(1: 2)$ ratio best discriminated among groups. Regression analyses further showed that tapping variability explained unique variance in word identification and spelling after controlling for phonological processing. These results suggest that efficient interhemispheric communication may facilitate the retrieval of lexical knowledge and thus contribute to reading ability independently of phonological processing.
\end{abstract}




\section{Acknowledgements}

My thanks and appreciation goes to Dr. Guy Lacroix for his unwavering support, guidance and encouragement. At our first meeting you gave me one of your many philosophical quotes about being "a good writer, in-becoming." This piece of encouragement meant a great deal to me at that time and is something that I will never forget. You have a natural teaching ability that is enhanced with your patience, humour, endless advice and energy. I above all thank-you for your unconditional support and dedication in helping me become confident in my skills and for helping me reach my goals.

I would also like to thank my volunteers especially those individuals with a learning disability and to Laura Brawn and Nancy McIntyre of the PMC for their continuous help with recruitment. Also, thank you to Tarra Anderson who was so valuable to multiple aspects of the study. I also had the great fortune of receiving thoughtful feedback and guidance from Dr. D'Angiulli, Dr. LeFevre, Dr. Hirotani and Dr. Campbell.

The support of my family throughout the years is the foundation of all my academic accomplishments. I especially want to thank my mom who gave me the encouragement and skills to pursue my dreams. I also want to thank Mike, who gave me infinite comfort, patience, and support. Finally, to Deanna, Gal, Lindsay, and Sabrina, thank-you for your friendship, guidance, mentorship, and the many laughs. 


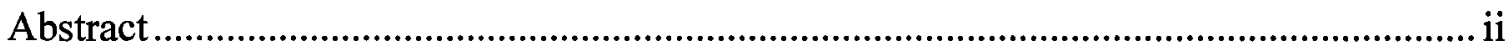

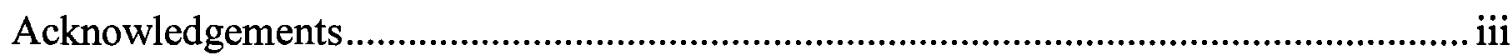

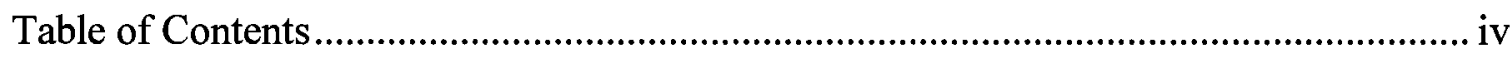

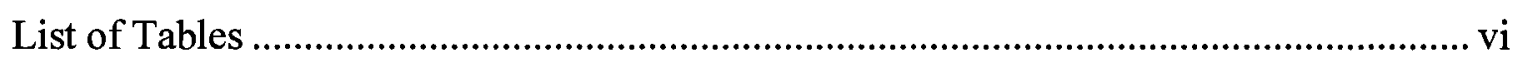

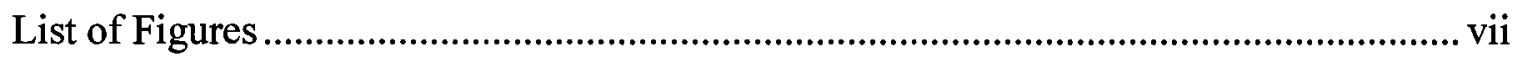

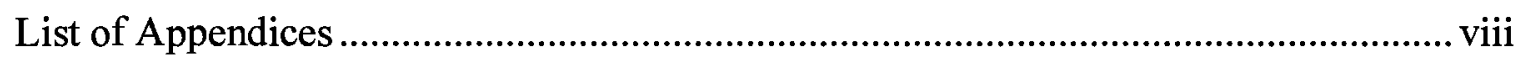

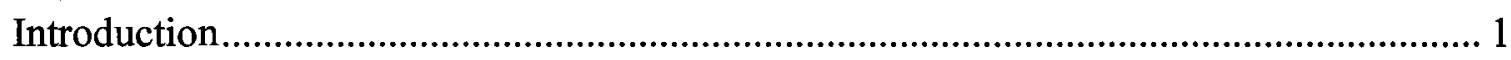

Learning Disabilities and Dyslexia ................................................................ 3

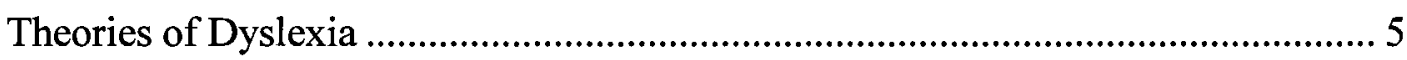

The Proposed Relationship Between Language and Bimanual Motor Movement ... 9

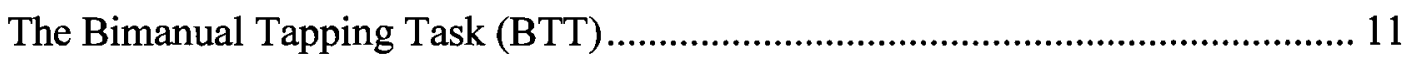

Critical Review of the Bimanual Tapping Task and Dyslexia............................ 16

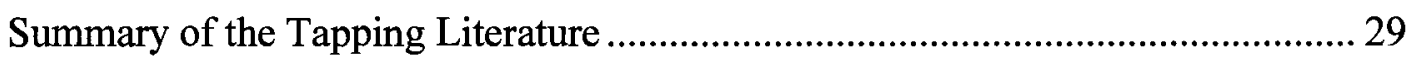

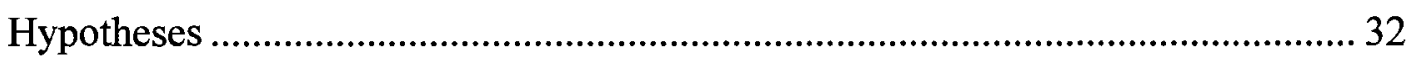

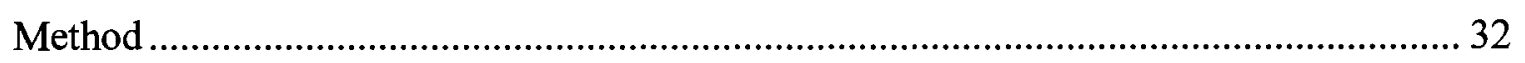

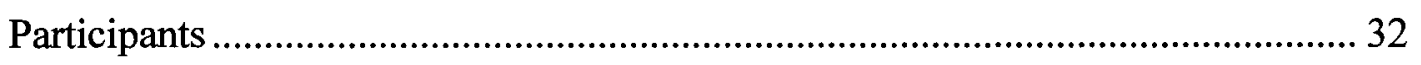

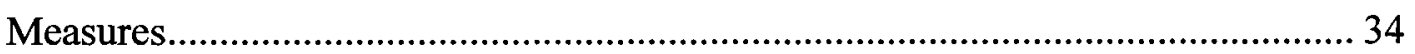

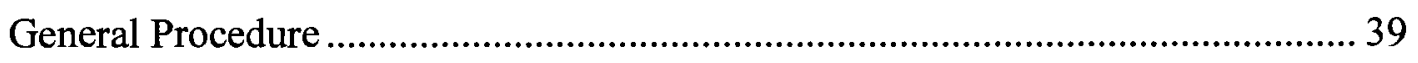

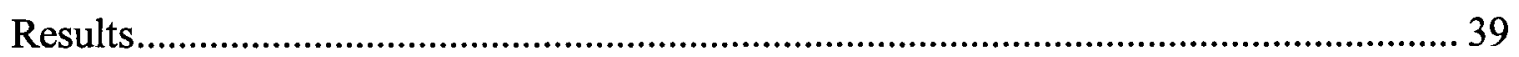

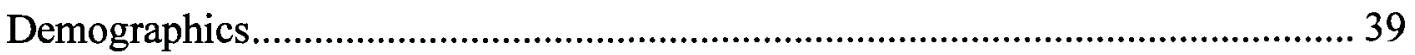

Measures of IQ, Working Memory, Phonological processing, Rapid Naming,

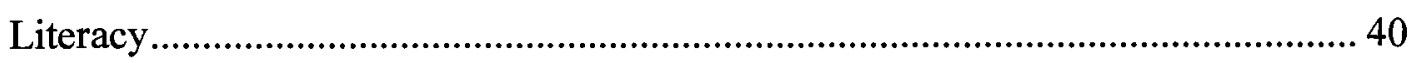


Bimanual Tapping Task Analyses............................................................................ 44

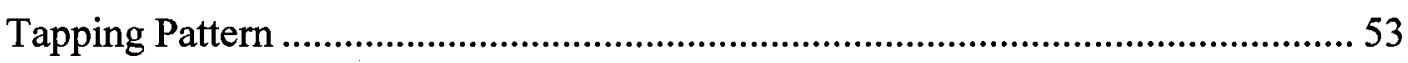

Correlations among the Variables ........................................................................... 54

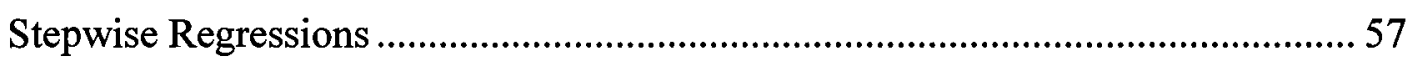

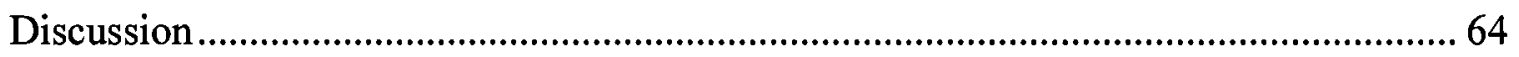

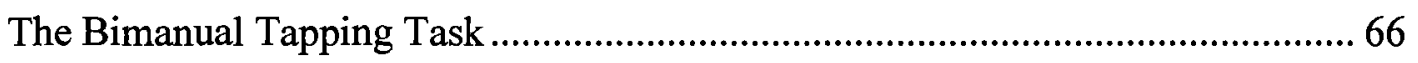

The Relationship Between Tapping and Reading ................................................. 68

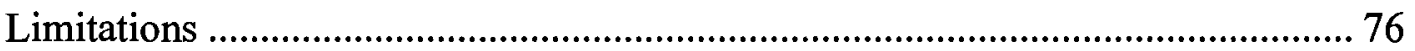

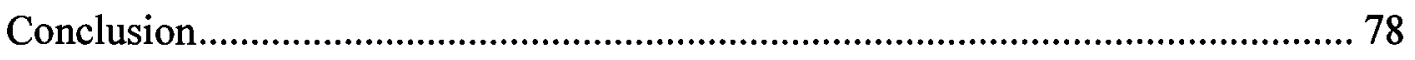

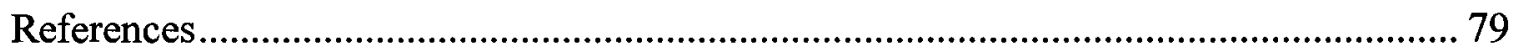

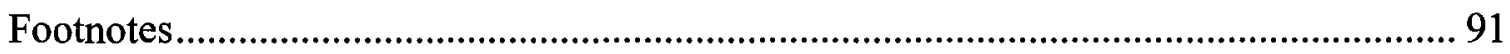




\section{List of Tables}

Table 1 Conversion chart of the three main units of measurement used to denote speed of tapping. 13

Table 2 Demographic information for Control $(n=59)$, Dyslexic $(n=25)$, and $L D / A D H D(n=11)$ groups 40

Table 3 Group comparisons for intelligence, literacy, phonological processing and

memory.

Table 4 Inter-correlation among reading measures and measures testing phonological processing, rapid naming, and working memory $(N=95)$.

Table 5 Correlation between Literacy variables and tapping variability for the 1:2 ratio at 184 BPM for the left and right fingers. 58

Table 6 Stepwise regression analysis predicting adults' WJ-III Word Attack performance. 60

Table 7 Stepwise regression analysis predicting adults' WJ-III Word Identification performance.

Table 8 Stepwise regression analysis predicting adults' WRAT Spelling performance... 62 Table 9 Stepwise regression analysis predicting adults' WJ-III Reading Fluency performance 63

Table 10 Stepwise regression analysis predicting adults' WJ-III Passage Comprehension performance. 64 


\section{List of Figures}

Figure 1. Vertical bars represent the metronome. Black circles represent the left finger responses while white circles represent the right. In the Asymmetrical conditions one finger responds twice as often as the other (adapted from Semjen \& Summers, 2002)... 15

Figure 2. Right hand tapping variability (ms) by Task (1:1 simultaneous, 1:2 ratio) and Group (Control, DD, LD/ADHD) collapsed across Speed with (SE bars)

Figure 3. Right hand tapping average (ms) as a function of Task $(1: 1$ simultaneous, $1: 2$ ratio) by Group (Control, DD, LD/ADHD) at the slow Speed (120 BPM) with (SE bars)

Figure 4. Right hand tapping average (ms) as a function of Task (1:1 simultaneous, 1:2 ratio) by Group (Control, DD, LD/ADHD) at the fast Speed (184 BPM) with (SE bars) 48

Figure 5. Left hand tapping variability (ms) as a function of Task (1:1 alternation and 1:2 ratio) by Speed (120 BPM, 184 BPM) collapsed across Groups with (SE bars).............. 50

Figure 6. Left hand tapping variability (ms) as a function of Task (1:1 alternation, 1:2 ratio) by Group (Control, DD, LD/ADHD) collapsed across Speed with (SE bars) ........ 51 Figure 7. Left hand tapping average (ms) as a function of Task (1:1 alternation, $1: 2$ ratio) by Group (control, DD, LD/ADHD) at the slow Speed (120 BPM) with (SE bars) ........ 52 Figure 8. Left hand tapping average (ms) as a function of Task (1:1 alternation, 1:2 ratio) by Group (Control, DD, LD/ADHD) at the fast Speed (184 BPM) with (SE bars) ........ 52 


\section{List of Appendices}

Appendix A-Group means and standard errors for tapping variability and tapping

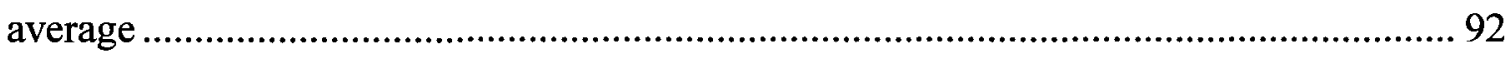




\section{AN INVESTIGATION OF READING AND BIMANUAL TAPPING ABILITY IN ADULTS WITH DYSLEXIA}

\section{Jessica Gunnell}

The majority of adults can process written information with ease and communicate with others using a variety of written media including text-messaging and e-mail. Some adults, however, have severe literacy problems that cannot be attributed to low intelligence, poor motivation, or lack of educational opportunities (Diagnostic and Statistical Manual of Mental Disorders Text - Revised [DSM-R], 2000). These individuals are generally diagnosed with dyslexia and their reading and spelling deficits have a negative impact on their daily lives.

Theories of dyslexia typically emphasize two different, but not necessarily mutually exclusive explanations of reading impairments: linguistic-cognitive theories and sensory/perceptual theories (Galaburda, 1993; Habib, 2000; Ramus, 2004; Ramus, Rosen, Dakin, Day, Castello, \& White, 2003; Smith-Spark \& Fisk, 2007; White, Frith, Milne, Rosen, Swettenham, \& Ramus, 2006; White, Milne, Rosen, Hansen, Swettenham, Frith, et al., 2006). Linguistic-cognitive theories suggest that reading difficulties are caused by phonological processing deficits. Researchers who support this position contend that problems with word/sound representations constitute the core deficit that underlies reading impairment in dyslexia (Ramus, 2004; Snowling, 1998; Snowling, 2001; Stanovich, 2005). In contrast, researchers who focus on sensory/perceptual theories examine the possibility that biological factors underlying perception and low-level cognitive processes in combination with phonological processing deficits yield a complete explanation of reading impairments (Au \& Lovegrove, 2001; Galaburda, 1993; 
Habib, 2000; Stein, 2001; Wolff, 1993). Researchers in this stream contend that language problems are a "surface expression of a deeper biological dysfunction" (Wolff, 1993, p. 87). Proponents of the sensory/perceptual theories argue that individuals with dyslexia typically present clinical characteristics that are unrelated to reading, including motor coordination problems (Badian \& Wolff, 1977; Gladstone, Best, \& Davidson, 1989; Fawcett \& Nicolson, 1992; Wolff, 2002; Wolff, Michel, Ovrut, \& Drake, 1990), letter reversals, left-right confusion (Miles, Wheeler, \& Haslum, 2003), memory problems (Helland \& Asbjornsen, 2004), and temporal/sequential processing difficulties (Stein, 2001). These "... 'extra-phonological' sources of variation" (Chiappe, Stringer, Siegel, \& Stanovich, 2002, p. 74) form the basis of the sensory/perceptual theories of dyslexia. The Temporal Processing Theory (Tallal, 1984) is a prime example of a theory that can be categorized as a sensory/perceptual theory (Habib, 2000).

The Temporal Processing Theory predicts that individuals with dyslexia differ from non-dyslexics in the temporal processing of visual, auditory, and motor events (for a review, see Farmer \& Klein, 1995). Proponents of the Temporal Processing Theory (e.g. Habib, 2000) maintain that researchers should investigate both linguistic and nonlinguistic symptoms. One method that has been employed to examine temporal processing is the bimanual rhythmic finger tapping task (Wolff, 1993; Wolff et al., 1990; Rousselle \& Wolff, 1991).

The Bimanual Tapping Task (BTT) requires coordinating the right and left index fingers to tap to an external beat. Wolff and colleagues have demonstrated that individuals with dyslexia tap with more variability and often commit more tapping errors than non-dyslexics (Badian \& Wolff, 1977; Wolff, Cohen, \& Drake, 1984). In addition, 
tapping is also known to correlate with spelling (Wolff, Melngailis, \& Kotwica, 1996; Wolff, Melngailis, Obregon, \& Bedrosian, 1995) and single word reading (Thomson, Fryer, Maltby, \& Goswami, 2006; Wolff et al., 1984). Wolff's findings are not without critics, however, and recent studies have cast doubts on his findings (e.g., Chiappe et al., 2002; Ramus et al., 2003). Therefore, this thesis had two goals. First, I attempted to replicate and extend Wolff and his colleagues' work and establish the tapping conditions that are most sensitive to differences among individuals with and without dyslexia. Second, I systematically examined how bimanual motor movement relates to different aspects of reading (i.e., decoding, word identification, comprehension, and fluency). Thus, this thesis is an empirical investigation of the relation between the temporal synchronization of action (i.e., bimanual motor movement) and dyslexia as assessed by bimanual finger tapping. The following sections begin review definitions of dyslexia, theories of dyslexia, the bimanual tapping paradigm, and critical studies that have investigated the relation between tapping and dyslexia.

\section{Learning Disabilities and Dyslexia}

Dyslexia is a specific Learning Disability (DSM-R, 2000; Learning Disabilities Association of Ontario [LDAO], 2001), affecting approximately 8 percent of Canadians between the ages of 16 to 55 ("Standing Committee," 2003). More specifically, dyslexia is defined as a "language-based disorder of constitutional origin characterized by difficulties in single word decoding, usually reflecting insufficient phonological processing abilities" (Lyon, 1995, p. 10 see also Lyon, Shaywitz, \& Shaywitz, 2003). Dyslexia is distinct from other forms of reading difficulties. It is characterized as a problem with single word identification that stems from a phonological processing deficit 
(Mapou, 2008). By comparison, a reading disorder is defined by impairments in reading fluency and reading comprehension. Mapou (2008) explains that most reading disorders are caused by phonological processing deficits. Nevertheless, a reading disability can be diagnosed even if phonological processing and word reading are not impaired.

Diagnosing dyslexia follows the same standards used for diagnosing a learning disability (LD). Since the 1960 's, the most referred to diagnostic criterion for an LD has been a discrepancy between intelligence (which is at least average) and academic achievement (LDAO; 2002; Lyon, 1996). Current diagnostic definitions of an LD have been expanded to include evidence of 1) impairments in one or more aspect of learning despite otherwise average abilities as well as 2) unexpectedly low achievement or 3) achievement that is only maintained by an abnormally high amount of effort (and outside support) (LDAO, 2002). Stanovich (2005) has made a strong argument for abandoning the intelligence discrepancy criterion, because it is not supported by empirical research. First, Stanovich argues that the primary difficulty for severely impaired readers is word recognition. Second, the evidence suggests that weak phonological processing skills (and their related physiological causes) lead to poor word recognition skills. Third, both phonological processing and word recognition difficulties do improve with remediation. Finally, neither phonological processing, nor word identification correlates with IQ (Stanovich, 2005, see also Stanovich, 1993). Nevertheless, no "gold standard" exists for defining or diagnosing dyslexia (Mapou, 2008). As a result, multiple definitions of dyslexia are found in the literature concerning reading impairments.

Following Chiappe et al. (2002) and Shaywitz et al. (2007), I operationalized dyslexia by focusing on two key characteristics in the present study: impaired 
phonological processing and word recognition skills. That is, the sample of participants with dyslexia in this thesis performed below the $26^{\text {th }}$ percentile on tests of word recognition and pseudoword reading.

Theories of Dyslexia

In reviewing the literature on dyslexia, Rice and Brooke (2004) concluded that despite decades of research, the field remains complex, speculative, and controversial. Part of this problem is a result of the heterogeneity of symptoms associated with dyslexia (Ramus, 2004). As a consequence, numerous theoretical frameworks have been developed to examine the complexities of the disorder (Habib, 2000). The main theories reviewed here are the Phonological Processing Theory, the Dyslexic Automatisation Deficit (Nicolson \& Fawcett, 1990), and the Temporal Processing Theory (Tallal, 1984).

The most widely accepted theory of dyslexia is the Phonological Processing Deficit Theory (Ramus, 2004; Stanovich, 1993). A consensus exists that the underdeveloped reading skills of individuals with dyslexia are largely due to early problems in phonological awareness (Snowling \& Hayiou-Thomas, 2006). Phonological awareness refers to the conscious awareness that words are characterized by particular sounds (i.e., phonemes) and that those sounds correspond to graphemes (i.e., written representations of phonemes). According to the phonological processing model, dyslexia is caused by a deficit at the level of phoneme representations (Habib, 2000). Thus, according to this model, individuals with dyslexia have difficulty representing, storing, and retrieving speech sounds from memory. Consequently, children and adults with dyslexia experience difficulty with phonologically related tasks such as phoneme deletion (e.g., Elision; pronounce book without the b), rhyming (e.g., which word does not fit: sit, 
hit, pit, fin?), and the retrieval of stored pronunciations of highly familiar words or symbols (e.g., rapid automatic naming (RAN), rapidly naming stimuli such as pictures, colors, letters or numbers) (Savage, 2004). Problems with phonological processing result in an insufficient ability to decode (i.e., interpreting letters in a meaningful way) or encode print (i.e., expressing oral words into written words).

Proponents of the Phonological Processing Theory maintain that poor phonological awareness is the cognitive deficit that accounts for much of the reading failure in individuals with dyslexia. Detractors of this theory do not dispute the fact that the majority of individuals with dyslexia have phonologically-driven reading difficulties. Instead, they argue that phonological deficits are an extension (or ostensible manifestation) of a more general problem and less specific sensorimotor dysfunction in the visual system, auditory system, the cerebellum, or temporal processing (Farmer \& Klein, 1995; Nicolson \& Fawcett, 1990; Stein \& Walsh, 1984; Tallal, 1984; White, Milne, et al., 2006; Wolff, 1993; Wolff et al., 1990). As a result, alternative theories have arisen such as the Dyslexic Automatisation Deficit (Nicolson \& Fawcett, 1990), and the Temporal Processing Theory (Tallal, 1984; see also Farmer \& Klein). These two theories highlight the overwhelming evidence that deficits in phonological processing are causally related to reading, but challenge the specificity of the phonological deficit. That is, phonological deficits are hypothesized to result from a more general impairment in sensorimotor domains (Ramus, Pidgeon, \& Frith, 2003).

Nicolson and Fawcett (1990) put forth the Dyslexic Automatisation Deficit Theory (DAD) and claim that dyslexia is not limited to reading problems. They hypothesize that dyslexia is caused by an underlying deficit in making any skill automatic 
(e.g., reading, driving, coordinated movement; for a review, see Nicolson, Fawcett, \& Dean, 2001 and Nicolson \& Fawcett, 1990). The cerebellum is the brain region that is purported to be associated with this deficit. Therefore, the DAD has also been referred to as the Cerebellar Theory. In addition, Nicolson and Fawcett (1990) theorize that abnormalities in the cerebellum are reflected in variety of behavioural measures that include phonological awareness, motor coordination, and reading and writing skills (Ramus et al., 2003). According to Nicolson and Fawcett, performance on all tasks, not just those related to reading, requires individuals with dyslexia to work harder than other individuals to allocate attentional resources to the task at hand. This is referred to as conscious compensation and explains why individuals with dyslexia can achieve normal performance on a wide variety of tasks. Poor performances of children with dyslexia in dual-task paradigms have been used as evidence to support the DAD theory (Fawcett \& Nicolson, 1992). When attention must be divided between two tasks, conscious compensation presumably becomes a less efficient strategy. Evidence to support deficits in automaticity has been inconsistent (see Savage, 2004).

An alternative sensory/perceptual theory is the Temporal Processing Theory (TPT) (for a review see Farmer \& Klein, 1995; but see also Studdert-Kennedy \& Mody, 1995). The term temporal is an abstract concept that relates to the way humans process time or generate timed events (Mauk \& Buonomano, 2004). Studdert-Kennedy and Mody (1995) define temporal processing as the ability to perceive temporal properties of an event (i.e., duration, sequence, and rhythm). A deficit in the ability to perceive temporal properties of an event is different from theories investigating deficit in processing perceptual information as would be hypothesized by the Magnocellular Deficit theory 
(see Stein \& Welsh, 1984 for a review). According to proponents of the TPT, individuals with dyslexia either have a deficit in the rate at which information is processed or have a deficit in processing certain aspects of an event (Chiappe et al., 2002). However, the TPT is not specific to visual and auditory processing of temporal events. Deficits in temporal processing may also affect the temporal organization of action (e.g., Wolff et al., 1990). Therefore, visual, auditory, and motor coordination problems are theorized to indicate an underlying deficit in temporal processing or temporal timing (Chiappe et al., 2002). Although some researchers identify temporal processing deficits with phonological processing deficits (i.e., Farmer \& Klein, 1995), others argue that impaired temporal processing constitutes an independent deficit and may be an additional cause of dyslexia (i.e., Wolff, 1993). The temporal organization of action (i.e., bimanual motor movement) will be the focus of the proposed research in this thesis.

In the literacy literature, the most frequently used indicator of temporal processing ability (in terms of action) has been bimanual motor coordination. Bimanual motor coordination refers to the coordination between the right and left sides of the body and has specifically been examined in the hands and fingers. For example, motor coordination tests such as the Bimanual Coordination Task (BCT, Gladstone et al., 1989) and the Finger Localization Task (FLT, Geffen, Nilsson, Quinn, \& Teng, 1985) have revealed that individuals with dyslexia have specific difficulties maintaining fluid movements and reproducing sequences with both hands (e.g., Gladstone et al., 1989; Moore, Brown, Markee, \& Theberge, 1995; Moore, Brown, Markee, Theberge, \& Zvi, 1996). The bimanual coordination task used in this research will be the Bimanual Tapping Task 
(BTT; Badian \& Wolff, 1977; Chiappe et al., 2002; Ramus et al., 2003; Wolff et al., 1990; Wolff et al., 1996; Wolff et al., 1995).

In a bimanual finger tapping task, participants coordinate the right and left index fingers to tap a pre-specified ratio to an external cue such as a metronome. For example, a one-to-two ratio (i.e., 1:2) requires the right hand to tap to every beat of the metronome, whereas, the left hand taps to every other beat. This type of motor sequencing is theorized to depend on the precise timing of rapid temporal sequential events (Wolff, 1993). Essentially, Wolff and colleagues argue that language and bimanual motor movements share the same principles of temporal organization and may be useful as an objective measure of timing precision, temporal organization, and processing (Rousselle \& Wolff, 1991).

The Proposed Relationship Between Language and Bimanual Motor Movement

The human brain is divided at the body midline into the right and left cerebral hemispheres. The corpus callosum, located in the white matter of the brain, is the largest fibre track that connects the two cerebral hemispheres (Bloom \& Hynd, 2005; Bogen, 2000). The corpus callosum is theorized to be the brain structure with the most influential role on interhemispheric communication. Interhemispheric communication or interhemispheric processing are generic terms referring to the exchanging or coordination of information between the two hemispheres (Banich \& Shenker, 1994).

The cerebral hemispheres have subtle anatomical asymmetries (Bloom \& Hynd, 2005). These subtle differences are assumed to play an important role in functional specialization, where the "right and left hemisphere have different roles in mediating various behaviours and higher mental processes" (Witelson, 1995, p. 61). Therefore, the 
two cerebral hemispheres can be conceptualized as independent but complementary systems each having some specialized processing functions (Bogen, 2000). Research has demonstrated that anatomical differences between the two hemispheres are responsible for the specialized functions that each performs (Damasio \& Geschwind, 1984). The most striking cerebral asymmetry is an area in the brain known as the planum temporal (PT), which is important for language (see Beaton, 1997 for an in-depth review). Interestingly, the PT asymmetry is hypothesized to be an evolutionary by-product of gesture comprehension (i.e., communication through motor movement) (Damasio \& Geschwind, 1984). Some neuroanatomical and behavioural findings also indicate that language processes overlap with the temporal organization of motor movement (Temple, Jeeves, \& Vilarroya, 1990). For example, the left hemisphere is one brain region that is associated with both language and bimanual motor movement (Wolff et al., 1984).

More recently, the cerebellum has been hypothesized to be a brain region related to language (Ivry, Justus, \& Middleton, 2001; Nicolson \& Fawcett, 1990; Nicolson et al., 2001) and is also known to be instrumental in timed bimanual motor movement (Ivry et al., 2001; Ivry \& Spencer, 2004; Ivry, Spencer, Zlaznik, \& Diedrichsen, 2002; Wing, 2001). Nicolson et al. (2001) suggested that a causal association between abnormal functioning of the cerebellum and reading dysfunction may exist. Nicolson et al. argued that cerebellum impairment would impoverish both bimanual motor movement and articulation, which, in turn, would affect phonological processing skills and thus cause impaired reading (but see also Ivry et al., 2001)

The corpus callosum has also been speculated to contribute to both language and bimanual motor movement. Neuroimaging studies (e.g., Robichon \& Habib, 1998; von 
Plessan et al. 2002) and postmortem exams studies (e.g., Galaburda, 1993) have found structural abnormalities in the corpus callosum in individuals with dyslexia. The corpus callosum is also instrumental in bimanual movement, however, its relationship with reading has been studied far less. Evidence that interhemispheric communication enhances reading ability has been developed by Weems and Zaidel (2004, 2005). They have shown that the left-hemisphere is superior at processing words compared to the right-hemisphere. Nevertheless, they have also shown that the quickest word recognition times (i.e., bilateral advantage) occur when both the right and left hemisphere have access to the word at the same time. This bilateral advantage is presumably enhanced by efficient interhemispheric communication.

In summary, serial motor coordination is theorized to share common cognitive processes with language. Wolff and colleagues suggest that a behaviour analysis of bimanual motor coordination can provide an avenue to examine impaired temporal organization without confounding individual differences in language. Moreover, the analysis of timing variables in motor coordination may shed light on some of the nonlinguistic impairments in dyslexia (Rousselle \& Wolff, 1991).

The Bimanual Tapping Task (BTT)

Rhythmic finger tapping typically requires participants to tap with their index finger to an external rhythmic signal and continue to tap at the same rate once the signal is turned off (Waber et al., 2000). Therefore, the BTT requires sensorimotor synchronization and reflects the ability to temporally coordinate a behavioural action with a reoccurring predictable external cue (Repp, 2005). The temporal coordination of serial motor action is theorized to be facilitated by one or more internal synchronization 
mechanisms (Semjen \& Summers, 2002). Based on this hypothesis, bimanual synchronization (i.e., two fingers tapping at the same time) and alternative-hand tapping (i.e., two fingers tapping in alteration of each other) occurs with reference to a single internal timekeeper. However, the limbs are prone to mutual influences (referred to generally as coupling) (Semjen \& Ivry, 2001; Semjen \& Summers, 2002).

The ability to synchronize tapping to an external cue (typically, a metronome) depends on the ability to predict future events (Repp, 2005). Although it would appear that an individual is tapping according to the metronome, in actuality, the tap is timed to coincide with one's anticipation of the next tone. Thus, tapping has been found to occur slightly before the next external cue. The upper temporal threshold refers to the approximate threshold at which this anticipatory tendency is stable (Repp, 2006). The upper limit refers to the fastest pace, rather than the slowest speed, that a specific tapping sequence (i.e., ratio) can be maintained. The upper temporal threshold is important because it establishes at which point an individual can perceptually track event sequences and then carry out actions in accordance with those event sequences (Repp, 2003). The essence of the TPT is a deficit in temporal processing, which relates to the rhythm, duration, and sequence of an event. Investigating the temporal properties of an action with the BTT, therefore, is only useful if the speed of tapping is within the upper temporal threshold. The upper temporal threshold for an auditory stimulus is approximately $200 \mathrm{~ms}=5 \mathrm{~Hz}=300 \mathrm{BPM}(\mathrm{Repp}, 2006)$. If this temporal threshold is exceeded, one of two things happen: 1) tapping becomes a reaction, not an anticipated event; or 2) in the case of complex bimanual tapping patterns (discussed below) 
participants may unconsciously revert to a simple bimanual movement such as $(1: 1)$ alternating tapping (Repp, 2005).

Table 1

Conversion chart of the three main units of measurement used to denote speed of tapping.

\begin{tabular}{ccc}
\hline Beats Per Minute & Hertz & Milliseconds \\
\hline 60 & 1.00 & 1000 \\
80 & 1.33 & 750 \\
90 & 1.50 & 667 \\
92 & 1.53 & 652 \\
100 & 1.67 & 600 \\
120 & 2.00 & 500 \\
150 & 2.50 & 400 \\
180 & 3.00 & 333 \\
184 & 3.07 & 326 \\
$240^{*}$ & $4.00^{*}$ & $250^{*}$ \\
300 & 5.00 & 200 \\
\hline
\end{tabular}

* upper temporal threshold for an auditory stimulus

Based on the above discussion, the first important consideration in bimanual tapping is speed. The speed of tapping is controlled by an auditory metronome and is characterized by one of three units of measurement: a) Beats Per Minute (BPM) b) Hertz (Hz: cycle-per-sec), or c) milliseconds (ms). BPM refers to how many tones the metronome emits within one minute $(1 \mathrm{BPM}=60 \mathrm{~s}=60000 \mathrm{~ms})$. Hz refers to how many times the auditory sound will repeat itself per second (i.e., $1 \mathrm{~Hz}=1$ tone per second) and milliseconds refers to how many milliseconds are between each auditory tone. The use of three different units of speed complicates the literature because high numbers in both $\mathrm{Hz}$ and BPM corresponds to small numbers in milliseconds (Repp, 2006). For example, 
$300 \mathrm{BPM}=5 \mathrm{~Hz}=200 \mathrm{~ms}$ and conversely, lower BPM and $\mathrm{Hz}$ corresponds to larger numbers in milliseconds, $50 \mathrm{BPM}=.83 \mathrm{~Hz}=1200 \mathrm{~ms}(\mathrm{Repp}, 2006)$. Table 1 displays a conversion chart for the three common units of speed. The upper temporal threshold is the rate at which tapping can be reliably performed.

The second important consideration for bimanual tapping is the tapping pattern or ratio. Repp (2007) found that multi-frequency ratios are generally more difficult to perform accurately than simultaneous tapping or tapping in alteration. A multi-frequency ratio refers to $\mathrm{a}(1: \mathrm{N})$ cycle where one finger taps $\mathrm{N}$ times (i.e., $2,3,4)$ while the other finger taps only once per cycle (Semjen \& Vos, 2002). For example, a multi-frequency ratio of $(1: 2)$ would require the right hand to tap to every beat of the metronome, whereas the left hand (the slow hand) taps to every other beat (see Figure 1). The hand that responds once per cycle is referred to as the slow hand, whereas the hand that responds to every tone is referred to as the fast hand (Semjen \& Summers, 2002).

The BTT task that Wolff and colleagues use typically requires participants to synchronize their tapping (or to "pace it") for the first $15 \mathrm{~s}$ to a target speed (e.g., 120 BPM). When the signal stops, participants must continue tapping at the same target rate for another 30 seconds. The latter part of the performance is known as unpaced tapping. The inter-response-interval during the unpaced phase should "reproduce the target interval" (Wing, 2002, p. 8) and this is called the tapping average. The variability around the tapping average (i.e., tapping variability) reflects the consistency of the rhythmic movement. It is measured by calculating the standard deviation of the Inter-ResponseInterval (IRI). The literature relating the BTT and reading has focused chiefly on tapping variability (see Chiappe et al., 2002; Ramus et al., 2003; Thomson et al., 2006; Wolff et 
al., 1996; Wolff et al., 1995; Wolff et al. 1990). Consequently, tapping variability was the central variable examined in this thesis. Nevertheless, additional information relating to how the task was preformed by participants was obtained by examining the tapping average and the tapping ratio. The tapping average is the time interval (IRI) between each finger's successive tap and the timing ratio is the proportion of responses by the fast finger divided by the responses of the slow finger (Wolff, 1993; Wolff et al., 1990). The statistics for each finger are recorded separately (Ramus et al., 2003; Thomson et al., 2006; Wolff et al., 1990).

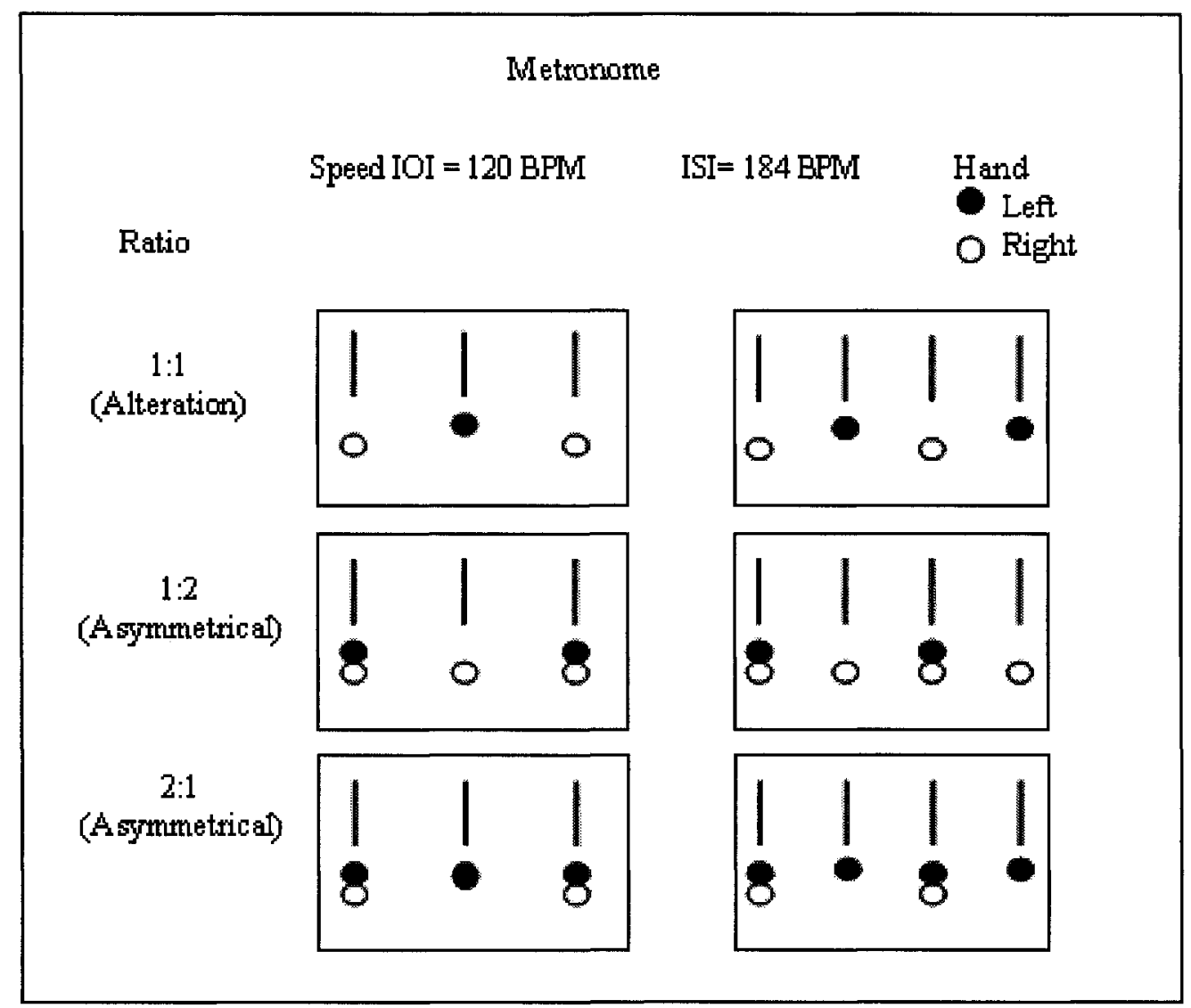

Figure 1. Vertical bars represent the metronome. Black circles represent the left finger responses while white circles represent the right. In the Asymmetrical conditions one finger responds twice as often as the other (adapted from Semjen \& Summers, 2002) 


\section{Critical Review of the Bimanual Tapping Task and Dyslexia}

This section begins with a review of Wolff and his colleagues' work on bimanual tapping in dyslexia. Wolff's early work is important because it emphasizes methodology (Badian \& Wolff, 1977; Rousselle \& Wolff, 1991; Wolff, 1993; Wolff et al., 1990). Wolff's later work has examined the relationship between selected aspects of literacy and tapping (Wolff et al., 1996; Wolff et al., 1995). The second portion of this section reviews the work of researchers who have adopted a critical perspective concerning the potential link between tapping and reading.

Badian and Wolff (1977) first used the BTT to examine motor sequencing skills in 12-year-old boys with reading disabilities $(\mathrm{RD})$. The $\mathrm{RD}$ group was reading below a standard score of 90 and had average IQs above 80 . The boys with an RD performed as well as younger boys without an $\mathrm{RD}$ on a single finger tapping task. When the boys tapped in alteration $(1: 1)$, however, the variability of tapping in boys with an RD was significantly greater compared to boys without an RD. In particular, the deterioration in tapping stability appeared more pronounced in the left hand of boys with an RD. The authors suggested that reading difficulties might be due to inefficient interhemispheric cooperation and this conclusion was supported by subsequent studies (e.g., Wolff et al., 1984).

Wolff et al. (1990) examined whether the timing deficit was developmentally stable, and consequently, observable in adults with dyslexia. They also sought to identify the conditions of the BTT that most efficiently distinguished control and dyslexic groups at different age levels. The focus of this review will be the results that pertain to the adult groups. The sample consisted of 41 adult dyslexics (mean age 22.6 years) with a mean 
full-scale IQ (FSIQ) of 107.7 and 41 age-matched adult controls, for whom the FSIQ had not been tested. Participants in the dyslexic group had FSIQ scores above 90 and were reading below the 9th grade level as assessed by the Gray Oral Reading Test (GORT). Wolff et al (1990) examined three interlimb coordination conditions: tapping in unison (0:1); tapping in alteration (1:1); and tapping asymmetrically and asynchronously (2:1). Additionally, three metronome speeds were tested: 92 BPM (i.e., $652 \mathrm{~ms}, 1.53 \mathrm{~Hz}$ ), 120 BPM (i.e., $500 \mathrm{~ms}, 2 \mathrm{~Hz}$ ), and 184 BPM (i.e., $326 \mathrm{~ms}, 3.07 \mathrm{~Hz}$ ). The results indicated that adult dyslexics and age-matched controls were comparable at all speed levels for both the alteration and unison conditions. In contrast, group differences in tapping variability were observed at the (1:2) ratio condition at 92 and 184 BPM. Additionally, approximately half of the dyslexic group and a few controls failed to maintain the correct tapping ratio of $1: 2$. The abnormal ratios were dominant (74\%) at $184 \mathrm{BPM}$ and appeared to result from the slow hand over-responding relative to the fast hand. A deviant tapping analysis was conducted to identify individuals who had a mean performance that was above one standard deviation from the normal control mean. The results revealed that approximately 50 percent of dyslexic participants and less than 10 percent of normal controls deviated from expected performances. In summary, Wolff et al. (1990) identified $184 \mathrm{BPM}$ at the $(1: 2)$ bimanual ratio as the most sensitive condition in discriminating between dyslexics and controls. The speed and condition that was least sensitive to group differences was $120 \mathrm{BPM}$ at both $1: 1$ alternation and 1:1 symmetrical.

Nevertheless, in a follow-up study, Rousselle and Wolff (1991) found that adults with dyslexia differed subtly from controls in a 1:1 simultaneous bimanual condition when individual tapping response patterns were analyzed longitudinally. In the 
simultaneous tapping condition all participants were expected to press the response buttons simultaneously with both fingers. That is, each finger was expected to make contact with its own respective button at the same time. Contrary to this prediction, one finger of all participants pressed the response button slightly before the other finger made contact. In this condition the right finger of the control group continuously pressed the response button slightly ahead of the left finger (despite instructions to have both fingers respond together). As a result, the tapping variability of the right finger was significantly smaller than the tapping variability of the left finger in control participants. This is referred to as a manual asymmetry because the right finger was more efficient than the left finger in tapping. In contrast, the dyslexic group did not show a lead finger preference and instead switched between the left and right fingers. As a result, the tapping variability of both fingers was not statistically different in participants with dyslexia. As a result, manual asymmetries on bimanual tapping were different between participants with and without dyslexia. Importantly, the difference in manual asymmetry was not evident with unimanual tapping (i.e., tapping with one finger). In both groups, a participant's preferred finger was more efficient at tapping than the non-preferred finger. Because unimanual tapping is restricted to one hemisphere (e.g., the left hemisphere controls bimanual motor movement on the right side of the body), the authors suggested that problems with bimanual coordination in individuals with dyslexia could not be limited to a timing problem in the left hemisphere. Rather, Rousselle and Wolff (1991) concluded that the timing deficits had to be related to a coordination difficulty involving both hemispheres. 
In the same study, Rousselle and Wolff (1991) also investigated phasic transitions. A phasic transition refers to the spontaneous change from a difficult tapping pattern (e.g., 1:2) to an easier tapping pattern (e.g., 1:1 simultaneous). Participants were required to tap a $(1: 2)$ ratio to an auditory metronome that continually increased in speed. As the metronome speed increased, the majority of participants eventually switched to either a synchronized or alternating tapping pattern (i.e., they executed a phasic transition). The data suggested that individuals with dyslexia experienced a phasic shift around 1.6 HZ (approximately: $96 \mathrm{BPM}$ or $625 \mathrm{~ms}$ ), whereas controls who shifted around 2.1 Hz (approximately: $127 \mathrm{BPM}$ or $427 \mathrm{~ms}$ ). This finding was important because it demonstrated the interaction between speed and ratio. To speculate, as speed increased, it may have been more difficult for individuals with dyslexia to inhibit unintended movements from the contralateral finger. Moreover, this effect became more pronounced when participants were required to tap a difficult ratio at a high speed.

In subsequent research, Wolff and his colleagues' (e.g., Wolff et al., 1996; Wolff et al., 1995) began to examine if impairments in bimanual tapping were related to specific aspects of literacy such as spelling and single word reading. Wolff et al. (1995) and Wolff et al. (1996) used the same cohort of participants. The two criteria to classify dyslexia were: a FSIQ of at least 100 and reading and spelling scores two years below grade level. Reading skills were assessed with the GORT and spelling skills were assessed with the Wide Range Achievement Test (WRAT). The bimanual tapping task conditions included: (1:1) alteration at 180, 240, and 300 BPM; and (1:2) ratio at 120, 150, and 240 BPM. Participants also completed RAN Letters, Colours and Objects (Denckla \& Rudel, 1976) and a motor speech task which required participants to repeat 
syllable strings in time with a different metronome speeds. Thus, the BTT, RAN and motor speech were used to measure timing and fluency abilities in dyslexia. Wolff et al.'s (1995) results suggested that 50 percent of dyslexics, regardless of age, had a deficit in timing control on all three measures of motor timing. Additionally, slower naming times for the RAN task were related to bimanual tapping ability in participants with dyslexia. Wolff et al. (1995) argued that the RAN task and the BTT may use similar processes to control timed behaviour. Thus, this relationship may identify a functional link between motor control, spoken language and reading impairments. Consequently, impaired temporal processing in motor coordination may be a plausible physiological cause of dyslexia.

However, recognizing that dyslexia is characterized by impairments in phonological processing, Wolff et al. (1995) and Wolff et al. (1996) investigated the possible relationship between phonological processing and bimanual motor coordination. Phonological processing was examined in a non-traditional way via the examination of spelling error type: dysphonetic or dyseidetic. Dysphonetic spelling refers to spelling by sight rather than by sound (Boder, 1973). For example, if the word is known to the individual who commits dysphonetic spelling errors, that individual may write something that resembles the target word but that has extraneous letters or syllables (e.g., faree for farther). If the word is unknown to that individual (i.e., not in the sight word vocabulary), the resulting spelling might be unintelligible to a reader if the target word cannot be ascertained (i.e., coetere for characters). Dyseidetic spellers spell the majority of words phonetically (e.g., laf for laugh) (Boder, 1973). Participants whose spelling errors were classified as dyseidetic were hypothesized to have good phonetic skills whereas those 
whose spelling errors were classified as dysphonetic were hypothesized to have impairments in phonetic skills. In his first study, Wolff et al. (1995) found that individuals with dyslexia who had a motor coordination deficit were 70 percent more likely to commit dysphonetic spelling errors compared to individuals who did not have motor coordination problems. In contrast, individuals with dyslexia who did not have motor coordination deficits tended to make more dyseidetic spelling errors. Moreover, in his second study, Wolff et al. (1996) found that motor coordination deficits accounted for 13 percent of the overall variance in dysphonetic spelling errors. Thus, Wolff et al (1995) and Wolff et al (1996) theorized that temporal resolution as assessed by motor coordination may have a causal link to some language skills such as spelling and phonology.

Notwithstanding Wolff et al. (1995) and Wolff et al. (1996), it can be argued that the biggest limitation in evaluating the validity of the TPT as an explanation of dyslexia is that few studies have comprehensively examined the relationship between temporal processing and specific components of reading ability (i.e., decoding, spelling, reading rate and comprehension) in relation to other cognitive factors. For instance, tapping deficits could potentially be attributed to deficits in other cognitive abilities, such as attention and memory, which Wolff and colleagues have not controlled for in their experiments. Therefore, the remaining part of this section will focus specifically on the relationship between tapping, different components of reading, and cognitive factors. This review will present competing explanations of Wolff and his colleagues' results.

Ramus et al. (2003) examined phonological, visual, auditory, and motor processing deficits in dyslexia. The BTT was used to examine bimanual motor 
movement. The sample included 16 dyslexics with a mean Full Scale intelligence quotient (FSIQ) of 122.7 (mean age 21.1 years) and 16 control participants with a FSIQ of 124.8 (mean age 21.9 years). The inclusionary criteria for dyslexia were a psychoeducational assessment completed by a certified professional, a FSIQ greater than a standard score of 100, and reading and spelling scores below a standard score of 110 . Control participants had FSIQ greater than the standard score of 100 and had reading and spelling scores at or above the standard score of 100 .

Ramus et al. (2003) administered three bimanual tapping conditions: 1:1 alternation at $2 \mathrm{~Hz}(500 \mathrm{~ms} / 120 \mathrm{BPM}), 1: 1$ alternation at $5 \mathrm{~Hz}(200 \mathrm{~ms} / 300 \mathrm{BPM})$ and 1:2 ratio at $4 \mathrm{~Hz}(250 \mathrm{~ms} / 240 \mathrm{BPM})$. In contrast to Wolff et al.'s (1990) results, Ramus et al. did not find significant differences in tapping variability between adults with and without dyslexia for any of the tapping conditions. As a result, the three tapping conditions were transformed into one composite score (the bimanual variable), which also failed to significantly distinguish the reading groups. Ramus et al. then computed a composite variable that merged the performances for bimanual tapping with that of other motor tests (i.e, bead threading). On this composite measure it was found that only four out of 15 individuals with dyslexia obtained scores at least $1.65 \mathrm{SD}$ away from the control mean. Moreover, a stepwise regression revealed that the composite variable did not explain any unique variance in literacy scores once phonological processing and auditory factors had been controlled. The authors concluded that timing deficits likely did not account for problems in literacy.

A critical review of Ramus et al.'s (2003) work reveals a number of important limitations. First, recall that Repp $(2005,2006)$ identified that the upper temporal 
threshold for the BTT is $200 \mathrm{~ms}$ (or $5 \mathrm{~Hz}$ ). Thus, the speed of $4 \mathrm{~Hz}$ that was used in Ramus et al.'s study may have been too difficult for participants in both groups. This speculative statement is supported by the fact that Ramus et al.'s participants in all groups had a large standard deviation in the (1:2) tapping condition. Moreover, as tapping ratio was not examined, it is possible that participants reverted to a simpler tap ratio such as tapping in alternation to meet the speed demands (see Repp, 2005). Such participant behaviour would account for Ramus et al.'s inability to find group differences.

Secondly, the mean standard reading score in the dyslexic group was 103 and their mean standard spelling score was 95.6. In comparison, the standard reading score in the control group was 113.9 and the standard spelling score was 115.3 . Hence, notwithstanding the psychoeducational assessments of the individuals with dyslexia, it appears that Ramus et al. (2003) compared individuals with average reading and spelling skills to individuals with above average reading and spelling skills.

The last limitation of Ramus et al.'s (2003) study is that the authors combined measures associated with the DAD theory (i.e., bead threading) and the TPT (i.e., tapping) to form a composite variable. The BTT measures the ability to process a temporal event. However, Ramus et al. merged aspects of temporal timing measured by the BTT with aspects of motor ability. Thus, it is not clear whether timing deficits are unrelated to reading or whether pure motor deficits are unrelated to reading. Finally, Ramus et al.'s sample size $(\mathrm{N}=32)$ was rather small to conduct regressions and might have lacked power to detect a significant relation between reading ability and temporal processing. 
In another study, Thomson et al. (2006) focused specifically on the relationship between auditory and motor rhythm awareness and reading in adults. The participants in the dyslexic group had previous diagnoses of dyslexia according to a psychoeducational assessment ( $n=19$; mean age 22.3 years). The average verbal IQ in the dyslexic group was 123 and the average performance IQ was 117 , as assessed by the Wechsler's abbreviated scale of intelligence (WASI; Wechsler, 1999). The control group was comparable ( $n=20$; mean age 22.3 years) with a verbal IQ of 128 and a performance IQ of 121. Other areas assessed were verbal short-term memory using a digit span test (i.e., remembering orally presented numbers and orally repeating these numbers back in the same sequence), timing and automaticity with the RAN task, and phonological processing with a phoneme deletion task (i.e. pronouncing book without the " $b$ "). The tapping task required participants to tap with their left finger only $(1: 0)$ to three separate beats: 1.5, 2, $2.5 \mathrm{~Hz}$ (i.e., 90, 120, $150 \mathrm{BPMs}$ ). Tapping was paced for 10 seconds and unpaced for 10 seconds. The results indicated that individuals with dyslexia tapped with greater variability (i.e., the standard deviation of the time between each tap or IRI) during the paced condition at 1.5 and $2 \mathrm{~Hz}$ and during the unpaced condition at $2 \mathrm{~Hz}$. Correlations revealed that lower IRI variability in the unpaced $2 \mathrm{~Hz}$ condition was related to better number recall in the digit span test $r=-.50$. The Digit Span task is arguably a verbal short-term memory measure, although critics have suggested that it may also be a measure of phonological processing (see Baddeley, Gathercole, \& Papagno, 1998). In addition, low IRI variability in paced tapping at $2 \mathrm{~Hz}$ was correlated with better reading scores $r=-.53$. A fixed order multiple regression, which included auditory measures, 
revealed that IRI variability did not account for any unique variance in reading, spelling, or phoneme deletion.

Thomson et al.'s (2006) study revealed that unimanual tapping did not account for unique variance in literacy measures. Thomson et al., however, did not examine bimanual tapping. This is a serious limitation because Wolffs (1993) review indicated that a bimanual 1:2 ratio at a high speed was the most sensitive condition for discriminating adults with and without dyslexia. On the other hand, Thomson et al. did choose a speed that was within a sensitive temporal range (Repp, 2005, 2006). An important insight gained from Thomson et al.'s study is that digit span was related to tapping. Wolff and colleagues had not previously controlled for individual differences in memory. Theoretically, tracking temporal events and remembering these events would require memory (Wing, 2002). Consequently, differences in memory might explain the usual results found by Wolff and his colleagues.

Chiappe et al. (2002) also investigated the relationship between general timing deficits and reading as proposed by the TPT theory. Timing deficits and reading were examined in the visual, auditory, and motor modalities. They classified impaired readers as those who performed at or below the 25 th percentile on WRAT reading $(n=30$; mean age $=25$ ). A control group of 32 participants, reading above the 29th percentile, was matched to the dyslexic group for age. In addition, a group of children $(n=31)$ who were reading above the $30^{\text {the }}$ percentile on WRAT reading participated (mean age $=9$ ). Adult participants completed the WRAT reading, spelling, and arithmetic tests, the WoodcockJohnson (WJ) Word Identification subtask, the Nelson-Denny Reading comprehension task, and a series of tests from the WAIS-R (vocabulary, block design, digit span and 
digit symbol coding). A phoneme deletion task was also administered to assess phonological processing. Finally, a variety of timed tasks in each of three modalities, auditory, vision, and motor were administered. The BTT used required participants to tap in an alternating (1:1) ratio to both fast (180 BPM) and slow (90 BPM) speeds. Tapping variability (i.e., the standard deviation of the IRI), tapping error (i.e., the number of early or late taps), and tapping rates (i.e., tapping to the right speed) were measured for each hand and used as the dependent variables.

A group by speed repeated-measures ANOVA on tapping variability revealed a main effect showing that individuals with dyslexia tapped with greater variability at both the slow and fast speeds. In addition, the dyslexic group tapped significantly faster at the slow rate compared to controls. Chiappe et al. (2002) expected to find a Speed by Group interaction on the BTT, but did not. These results actually replicate Wolff et al.'s (1990) findings in that no interaction for group and speed was found for the alteration $(1: 1)$ tapping. In addition, Chiappe et al. also replicated the finding that dyslexics tapped with more variability than non-dyslexics at both fast and slow speeds. Note that the interaction in Wolff et al.'s (1990) experiment was found only when examining the difference between an asymmetrical ratio (1:2) and the (1:1) alteration ratio at a fast speed.

Nevertheless, correlations revealed that tapping variability was moderately related to reading, spelling and phonological processing. The relationship suggested that better readers were also better at maintaining a consistent IRI and thus were less variable tappers (range: $\mathrm{r}=-0.33$ to -0.45 ). After controlling for phonological processing, a hierarchical regression revealed that RAN was the only timing variable that accounted for unique variance in WRAT reading scores. Moreover, the RAN task also contributed 
unique variance to WJ-Word Identification after phonological processing had been partialed out. A commonality analysis was then performed to identify the unique and common variance between WRAT reading, phonological processing, naming speed (RAN), and tapping variability. Tapping variability accounted for $9.7 \%$ of the variance in WRAT reading, but only $.97 \%$ was unique. RAN and tapping variability did not share common variance in reading. In contrast, phoneme deletion accounted for $45 \%$ of the variance in WRAT reading. This can be decomposed into $19.6 \%$ unique variance, 16.7 $\%$ variance shared with RAN, $5 \%$ variance shared with tapping, and $3.8 \%$ shared with both RAN and tapping. According to Chiappe et al., (2002) the above results are indicative of a phonologically-based deficit.

The results from Chiappe et al.'s (2002) study cast substantial doubt on the TPT. To date, this study is one of the most comprehensive in examining timing variables and literacy correlates. A valuable feature in this study was the strict inclusionary criteria of the dyslexic participants. All participants with dyslexia were selected according to a current reading impairment. This is in contrast to Ramus et al. (2003) and Thomson et al. (2006) who predominantly used previous reports as inclusionary criterion for dyslexia. Another beneficial feature of Chiappe et al.'s (2002) study was the large sample size, which helps to strengthen the power of the regression results. In addition, the authors carefully examined the unique and shared variance between variables.

Chiappe et al.'s (2002) findings, conversely, cannot definitively rule out the possibility that temporal integration of asymmetrical bimanual motor movement may explain unique variance in reading ability. This is because a bimanual ratio, such as $(1: 2)$ was not administered. Correlations and regressions between tapping and reading should 
therefore be interpreted with caution. To speculate, a more difficult ratio that required asynchronous coordination of two hands may be a better predictor of literacy or academic skills than a simple (1:1) ratio. The important findings from Wolff and his colleagues' work was that motor deficits appear to be rate dependent (i.e., speed) and were more difficult when asynchronous integration of movement was required (Wolff et al., 1990).

The final study to be reviewed was conducted by Waber et al. (2000). Waber et al.'s sample included one hundred English-speaking children (age 7 to 11) referred to the study because of learning problems and 243 children without learning problems. Three tapping conditions were administered: 1) unimanual tapping (i.e., 0:1; 1:0) at 90 BPM, 2) (1:1) alteration at 180 and $240 \mathrm{BPM}$, and 3) (1:2) ratio at 120, 180 and 240 BPM. Zscores were computed for each of the above conditions and composite scores were developed for the tapping conditions administered at more than one speed. Results showed that tapping was less stable during the alternation and ratio tapping conditions for children with learning problems. However, increased tapping variability was not specific to children with documented learning problems. A regression analysis revealed that " 1 SD unit in tapping variability was associated with the loss of approximately 3 points in performance on the achievement tests" (p. 192). The magnitude of this effect was greatest in the bimanual (1:2) condition. Interestingly, poor academic achievement in single word reading, spelling, and math was related to greater tapping variability within both groups of children. These associations appeared reliable, but the authors noted that the magnitude of the effect was modest. The authors hypothesized that tapping variability may function as an indicator of the effectiveness of how information is being integrated and processed within the brain. Reading in particular may be sensitive to problems in 
temporal timing and integration because literacy skills are theorized to require fluency, integration, and precision of information within the brain (Waber et al., 2000).

This study is important because increased tapping variability on an asymmetrical ratio did appear to be related to single word reading and spelling. A major limitation of this study, in terms of relevance to the present study, was the use of children. Previous work has demonstrated that difficulty of the ratio (i.e., $0: 1,1: 1,1: 2)$ is influenced by age (see Wolff et al., 1990; Wolff et al., 1995). In addition, the tapping speed of 240 BPM was likely too high for children. This probably inflated the tapping variability in the composite scores. Another limiting feature is that Waber et al. (2000) did not control for phonological variables, or other timing variables such as RAN. Nevertheless, Waber et al.'s study is noteworthy because the BTT conditions were closest to those suggested by Wolff (1993) and that condition successfully predicted reading and spelling ability.

\section{Summary of the Tapping Literature}

According to proponents of the TPT, an individual with dyslexia processes temporal events such as duration, sequence, or rhythm differently than a non-dyslexic (Chiappe et al., 2002). In other words, individuals with dyslexia, according to the TPT, have difficulty with the temporal events that are derived from perception. Wolff and his colleagues" work have focused on the coordination of bimanual action, "with a particular emphasis on the temporal variables of timing precision and serial ordering" (Wolff, 1993; p. 89). This line of research is potentially fruitful because the temporal organization of action is theorized to share the same temporal organization of language. Thus, the BTT may provide a window of investigation, not into the motor system per se, but rather the cognitive architecture (Ivry, Diedrichsen, Spencer, Hazeltine, \& Semjen, 2004) of 
processing temporal events without the influence of individual differences in language. That is, because there is no obvious direct causal relationship between rhythmic finger tapping and reading/spelling, this research centers on the assumption that temporal processing as measured by bimanual coordination may overlap with the neural mechanisms of language processing (Waber et al., 2000; Wolff, 1993).

A critical issue in the tapping literature at the present time is whether the BTT can explain variance in reading that cannot be accounted for by phonological abilities or whether it simply underlies problems in phonological processing (Chiappe et al., 2002). Although Wolff and colleagues have identified the most sensitive BTT parameters (an asymmetrical 1:2 ratio at a speed of 184 BPM), the specific cognitive components that relate the BTT to reading are still relatively unknown. As a response to this shortcoming in the literature, Chiappe et al. (2002), Ramus et al. (2003), Thomson et al. (2006), and Waber et al. (2000) have endeavoured to address this critical shortcoming. Unfortunately, for the reasons discussed in the previous section, the BTT methodology used in these studies, as well as other difficulties, prevents one from drawing definitive conclusions. Nevertheless, these studies have strong methodological features that have been included in the present study.

First, Chiappe et al. (2002) had a well defined inclusionary criterion for dyslexia. In addition, they tested a large sample as was also the case in Waber et al.'s (2000) study. Second, Thomson et al.'s (2006) study included a measure of short-term memory (e.g., digit span). The study demonstrated that individuals with better number recall also tapped with less variability. Therefore, individual differences in short-term memory, and not necessarily temporal processing, could explain the tapping differences found by Wolff 
and colleagues. In the present study, a working memory span task (Counting Span) was used (Case, Kurland, \& Goldberg, 1982; Conway, Kane, Bunting, Hambrick, Wilhelm, \& Engle, 2005). Because the counting span task does not involve linguistic stimuli, it is ideal for samples with impaired language abilities. Third, another common strength in all the studies, with the exception of Waber et al.'s (2000) study, was the inclusion of a phonological processing task. A particular strength of Chiappe et al. (2002) was the examination of the unique variance that timing accounted for in phonological processing. This was an insightful choice because it allows one to examine whether a phonological deficit could arise from a deficit in temporal processing and, in this way, cause reading and spelling impairments. Finally, many of the reviewed studies included the RAN task. Although the exact nature of the RAN task is a matter of debate, including the RAN task is warranted. Whether it taps phonological processing as claimed by some authors (e.g., Ramus et al., 2003) or aspects of attention, memory, and timing fluency as claimed by others (e.g., Wolf, Bowers, \& Biddle, 2000), the RAN task is a consistent predictor of reading ability (Wolf et al., 2000).

Thus, the goal of this research was to re-evaluate the validity of the Temporal Processing Theory of dyslexia. The relation between the bimanual tapping task and reading was examined in an adult population. First, the BTT condition that Wolff et al. (1990) identified as the most diagnostic (i.e., 184 BPM at an asymmetrical 1:2 ratio) was used to confirm whether individuals with dyslexia tapped with greater variability than controls. In order to gain a better understanding of temporal processing and its relationship to reading, the present study also measured multiple aspects of literacy including decoding, single word identification, reading rate, comprehension, and spelling. 
Finally, individual differences in IQ, memory, and phonological processing were measured.

Hypotheses

First hypothesis. If the relation between the Bimanual Tapping Task and reading ability is reliable, then the proposed study will replicate Wolff et al.'s (1990) results. Hence, it is expected that participants with dyslexia will show greater tapping variability than the control participants when a high metronome speed (184 BPM) is used in conjunction with a difficult tapping ratio (1:2).

Second hypothesis. The temporal processing theory predicts that problems in reading, spelling, and phonological processing in dyslexia are an extension of an underlying problem in processing temporal events. The BTT examines the synchronization of action (i.e., tapping) that is organized through a rhythmic event (i.e., temporal processing). Furthermore, action is hypothesized to depend on the same structures as language. If the above explanation is accurate, then this proposed study expects that tapping at $184 \mathrm{BPM}$ in a $1: 2$ ratio condition will explain unique variance in decoding, single word reading, spelling, and reading fluency regardless of potential contributing factors such as working memory, IQ and phonological processing. Thus, better tappers (i.e., low tapping variability) will also score higher on measures of sight word reading, spelling, and reading fluency.

\section{Method}

\section{Participants}

Ninety-nine university students with English as a first language were tested. Four participants, however, were excluded from the final analyses: three participants' tapping 
scores where lost due to software problems and one participant was unable to complete the tapping task because of a hand tremor owing to a physical disability. Thus, the final sample included 95 participants. Participants were recruited from a first-year undergraduate psychology student participant pool and with advertisements posted on campus. In addition, a qualified learning specialist at the university center for students with disabilities e-mailed an advertisement to registered students whose records indicated below average skills in decoding and single word identification. Participants received extra marks towards their first-year psychology course or twenty dollars as compensation.

Participants were classified as dyslexic if their average percentile score on Word Identification and Word Attack was at or below the 26th percentile (Chiappe et al., 2002; Shaywitz et al., 2007). Twenty-five participants met this criterion and were assigned to the dyslexia group (DD). Seventeen of these participants had clinical documentation of an LD, reported a history of reading and academic difficulty, and all but one was registered with the Paul Menton Centre (PMC) for Students with Disabilities. Five participants who did not have a clinically diagnosed LD reported current and historical problems with reading. The remaining three participants did not report reading difficulties. One participant in the DD group also had clinically defined Attention-Deficit Hyperactivity Disorder (ADHD).

Eleven participants reported having an LD and or ADHD, but obtained an average score on Word Identification and Word Attack that was above the 26th percentile. Hence, these participants did not meet the criterion for dyslexia. Nevertheless, the presence of an LD and or ADHD excluded the possibility of assigning these participants to the control group because both disorders have an impact on higher order neurocognitive processes 
that involve tests of attention/concentration, executive functioning, working memory, processing speed, and motor speed (see Hervey, Epstein, \& Curry, 2004; LDAO, 2002). Thus, a Learning Disability/Attention-Deficit Hyperactivity Disorder group (LD/ADHD) was created. Ten participants reported having formal documentation of an LD and or ADHD by a clinical psychologist and were registered with the university center for students with disabilities. Specifically, three participants were diagnosed with an LD only, one participant was diagnosed with ADHD only, and six participants were diagnosed with both an LD and ADHD. The nine participants with a clinically diagnosed LD also reported a history of reading related problems. The remaining participant did not have clinical documentation of an LD. This participant believed he had an LD that affected spelling. Also, he reported current and historical difficulty with spelling, and reported receiving modified programming during elementary school. Considering that this participant's WRAT spelling score was in the 14th percentile, he was retained into the LD/ADHD group. Therefore, this group was heterogeneous in nature.

The remaining participants $(n=59)$ constituted the control group. The participants in this group achieved an average score on Word Identification and Word Attack that was above the 26th percentile and did not report an LD or ADHD.

\section{Measures}

Background questionnaire. The background questionnaire assessed participants' self-reported age in years, gender (male or female), preferred handedness (right, left or other), and academic background. Participants were asked to disclose current academic year (first, second, third, fourth, post-graduate, and other) and overall university grades in percent or letters. The letter grades were subsequently changed using Carleton's grading 
system to the first number of the letter range. For example, a grade Letter of A- (80 to 84 percent) was given a value of 80 . In addition, participants were asked to report their musical and athletic ability on a Likert scale ranging from 1 (well below average) to 5 (well above average).

Kaufman Brief Intelligence Test, Second Edition (KBIT-2; Kaufman \& Kaufman, 2004). Expressive vocabulary and matrices were used to obtain measures of verbal and nonverbal IQ respectively. The expressive vocabulary subtest consisted of 60 items. Participants were asked to point to one of several pictures that best represented a word given by the experimenter. The matrices subtest consisted of 46 items. Participants were asked to choose a picture that best completed a picture or sequences of abstract stimuli. Both subtests were discontinued after four consecutive wrong answers. The KBIT took approximately 20 to 25 minutes to administer. The raw scores for the expressive vocabulary subtest and the matrices subtests were converted into age based standard scores $(M=100 ; S D=15)$.

Woodcock-Johnson III Tests of Achievement (WJ-III; Woodcock et al., 2001). Four subtests from Form B were administered: Word Identification, Word Attack, Passage Comprehension, and Reading Fluency. Word Identification required participants to read real words aloud. Word Attack required participants to read a list of pseudowords and measured phonological and orthographical decoding. Passage Comprehension required participants to read a passage and provide one word that meaningfully completed a blank space in the text. Word Identification, Word Attack, and Passage Comprehension were discontinued after six consecutive wrong answers on a page. Reading Fluency required participants to read some sentences as quickly as possible and 
evaluate whether the sentence was true or false (e.g., "A cow is an animal Y or N"). Participants were given up to three minutes to complete the entire test which consisted of 98 sentences. One point was given for each correct response and time was recorded for participants who finished before three minutes. The WJ-III was normed on a large sample $(N>8000)$ and a wide range of age groups ( 2 to $90+$ years). The $\mathrm{WJ}$ also has good reliability (see Strauss et al., 2006). Raw scores were converted into age appropriate percentiles and standard scores $(M=100 ; S D=15)$.

Wide Range Achievement Test - 4 (WRAT; Wilkinson \& Robertson, 2006). The green form of the spelling subtest was administered. The spelling subtest assessed participants' ability to correctly write words from an orally dictated list. The spelling test consisted of 42 words and was discontinued after 10 consecutive spelling errors. The WRAT-4 was standardized in the United States on a sample of 3000 participants belonging to age groups ranging from 5 to 95 years. The reliability coefficient by age ranges between $.87-.93$. The raw scores were transformed to age normed standard scores. Comprehensive Test of Phonological Processing (CTOPP; Wagner, Torgesen, \& Roshotte, 1999). Four tests were administered: Elisions, Phoneme Reversal, RAN Letter and RAN Objects. Elisions consisted of 20 words. The task required participants to remove a pre-specified syllable from a word in order to produce a new word (e.g., "bold" without the /b/ to create "old"). Phoneme Reversal consisted of 18 pronounceable pseudowords. Participants were asked to pronounce the phonemes of the pseudoword in reverse order to produce a real word. (e.g. "em" to produce "me"). Both Elisions and Phoneme Reversal were discontinued if three consecutive errors were made. 
Both Forms A and B of RAN Letters were administered. Form A and B were on two separate pages in which the letters $(\mathrm{s}, \mathrm{t}, \mathrm{n}, \mathrm{a}, \mathrm{k}$ and $\mathrm{c}$ ) on each form were arranged in a 4 x 9 pattern. Additionally, both Forms A and B of RAN Objects were administered. The stimuli for RAN objects included pictorial representations of the following objects: pencil, star, fish, chair, boat and key arranged in a $4 \times 9$ pattern. Following the standardized instructions, participants were asked to name the letters or objects aloud as quickly as possible. In all cases, participants self-corrected if a mistake was made. The dependent measures were the sum of the naming time in seconds for RAN Letters form A and B and the sum on the naming time for RAN Objects form A and B.

Counting span. Working memory capacity was measured using a counting span task (Case, et al. 1982; Conway et al., 2005). Engle's (2007) version of this task was used (See also Unsworth, Heitz, Schrock, \& Engle, 2005) and was administered using the software E-prime (Schneider, Eschman, \& Zuccolotto, 2002a, 2002b). Participants viewed a series of slides on a computer screen that displayed variable numbers of dark blue circles, light blue circles, and dark blue squares. For each slide, participants were instructed to count the number of dark blue circles aloud and to remember the total. The experimenter monitored counting accuracy. On some occasions, when a participant obtained a mistaken count, the experimenter corrected the participant. After 2 to 6 object slides, participants were prompted to recall the totals from each slide in order. In total, fifteen series of slides were used (three for each of the following recall interval categories: $2,3,4,5$ and 6 ). The same pseudo-random order was used for all the participants. The participants also received three practice trials. Individual performances were computed using the partial-credit load scoring method (Conway et al., 2005). One 
point was counted for each dark blue circle total recalled in the correct serial position for each series of slides. The maximum score was 60 .

Bimanual Tapping Task (BTT). Hand Tapping Software Version 2.0 (Pitre, 2006) was used to run all the BTT conditions. The program was executed on a PC compatible computer running with the Windows XP operating system and a runtime version of Java of 1.5.0 or above. The program was written in Java and uses an external timer library (JNI). Participants' motor responses were recorded using a standard computer keyboard. At the start of this portion of the experiment, participants were seated facing a computer screen. Before each condition, the experimenter explained the tapping task to be accomplished, demonstrated the task, and asked participants to demonstrate the tapping pattern to ensure full comprehension of the task demands. Participants were then asked to place their left index finger on the left-hand Control key of the keyboard and their right index finger on the right-hand Control key. The experimenter started each trial by initiating the digital metronomic beat. Participants synchronized finger tapping to the metronome for 15 seconds (paced tapping) and continued to tap unaccompanied by the metronome for another 30 seconds (unpaced tapping). Thus, each trial lasted 45 seconds in total. Statistics were recorded only for unpaced tapping.

Participants completed three ratios: (1:1) synchronization, (1:1) alternation and $(1: 2)$ ratio at each of two speeds: $184 \mathrm{BPM}(326 \mathrm{~ms}=3.07 \mathrm{~Hz})$ and $120 \mathrm{BPM}(500 \mathrm{~ms}=$ $2 \mathrm{~Hz}$ ). The speed of $120 \mathrm{BPM}$ is referred to as Slow and the speed of 184 BPM is referred to as Fast. Finally, speed and ratio were counterbalanced across participants. For both the left and right fingers, the software calculated the total number of taps, the mean interval 
between each tap (i.e., average inter-response-interval (IRI)), and the standard deviation around the average IRI (i.e., tapping variability).

\section{General Procedure}

The experimenter first obtained informed and voluntary consent. The tests were administered in a single session ${ }^{1}$ and were presented in the following order: background questionnaire, KBIT-2, Elisions, Phoneme Reversal, RAN letters, RAN Objects, Reading Fluency, Passage Comprehension, Word Identification, Word Attack, tapping tasks, Counting Span, and Spelling. Participants were tested individually in a small and quiet room. The length of each session ranged between 100 and 120 minutes and breaks were provided as necessary. Once testing was completed, participants were thoroughly debriefed.

Results

\section{Demographics}

Demographic variables for the DD, LD/ADHD and control groups are presented in Table 2. Group difference for age, grades and academic standing were analyzed using a $1 \times 3$ between-subjects ANOVA. Group differences for musical ability and athletic ability were analyzed using a 1 X 3 Kruskal-Wallis ANOVA. A significant difference was found for age, $F(2,94)=11.40, p<.001$. A Sidak-adjusted post-hoc comparison revealed that participants in the LD/ADHD group were older $(M=28.82, S D=7.97)$ than those in both the Control $(M=21.32 ; S D=4.90)$ and DD groups $(M=21.56, S D=$ $2.42), p s<.001$. The remaining analyses showed that the groups did not differ in grades, $F(2,94)=.29, p=.75$, academic standing, $F(2,94)=1.21, p=.30$, musical ability, $\chi^{2}$ $(2, N=95)=1.99, p=.37$, or athletic ability, $\chi^{2}(2, N=95)=.525, p=.77$. 
Chi-Square analyses could not be conducted to analyze Gender and Handedness because both contingency tables contained at least one cell with a count smaller than five. The descriptive statistics suggest that the proportion of males was somewhat higher in the control group (37\%) than it was in the DD group (25\%) and the LD/ADHD group (22\%). The descriptive statistics also unexpectedly show a much higher proportion of lefthanded participants in the control group (23\%) than in the DD group (4\%) or LD/ADHD group $(10 \%)$.

Table 2

Demographic information for Control $(n=59)$, Dyslexic $(n=25)$, and LD/ADHD $(n=$ 11) groups.

\begin{tabular}{|c|c|c|c|c|c|c|c|c|c|c|c|}
\hline & \multicolumn{2}{|c|}{ Gender } & \multicolumn{2}{|c|}{ Hand } & \multicolumn{2}{|c|}{ Age } & \multirow{2}{*}{$\begin{array}{l}\text { Year } \\
M d n\end{array}$} & \multicolumn{2}{|c|}{ Grades } & \multirow{2}{*}{$\frac{\text { Music }}{M d n}$} & \multirow{2}{*}{$\frac{\text { Athl }}{M d n}$} \\
\hline & $\mathbf{M}$ & $\mathrm{F}$ & $\mathrm{R}$ & $\mathrm{L}$ & $M$ & $S D$ & & $M$ & $S D$ & & \\
\hline Control & 16 & 43 & 48 & 11 & 21.32 & 4.90 & $2^{\text {nd }}$ & 75.69 & 7.34 & 3 & 3 \\
\hline Dyslexic & 5 & 20 & 24 & 1 & 21.56 & 2.42 & $3^{\text {rd }}$ & 74.60 & 7.99 & 2 & 4 \\
\hline LD/ADHD & 2 & 9 & 10 & 1 & 28.82 & 7.97 & $3^{\text {rd }}$ & 74.24 & 7.45 & 3 & 4 \\
\hline
\end{tabular}

Notes: Hand = hand preference; Music = musical ability; Athl = athletic ability.

Measures of IQ, Working Memory, Phonological processing, Rapid Naming, Literacy

The descriptive statistics for intelligence (Verbal and Nonverbal), working memory (Counting Span), phonological processing (Elisions and Phoneme Reversal), rapid naming (Object and Letter), and literacy (Word Identification, Word Attack, Passage Comprehension, Reading Fluency, and Spelling) by groups are given in Table 3. The means (and standard deviations) for Verbal and Non-verbal IQ, Word Identification, Word Attack, Passage Comprehension, Reading Fluency and Spelling are age appropriate standard scores. Age norms were not available for the remaining measures. Thus, the 
means and standard deviations of the raw scores are given for Counting Span, Elisions, Phoneme reversal, RAN Objects and RAN Letters (see Table 3).

In the previous section, it was found that there were significant differences in age among the groups. Although seven measures had normative data that adjusted for age (i.e., KBIT, WJ, and WRAT), the remaining five measures did not (i.e., Counting Span, Elisions, Phoneme Reversal, RAN Objects and Letters). Consequently, for these five measures, residual scores adjusted for age were used for final analyses.

To establish that participants in the DD group had significantly lower scores for Word Identification and Word Attack, and to ascertain any other possible differences among the groups, $1 \times 3$ between-subjects ANOVAs were conducted for all the measures. However, Levene's test for equality of variances showed that the assumption of homogeneity of variances was violated for Word Identification, $F(2,92)=5.01, p=.009$, Word Attack, $F(2,92)=4.84, p=.01$, Elisions, $F(2,92)=8.33, p<.001$, RAN Objects, $F(2,92)=4.39, p=.02$, and RAN Letters $F(2,92)=5.88, p=.004$. Therefore, for the measures that violated the assumption of homogeneity of variance, Welch's varianceweighted ANOVA was used to test between-subject effects. 

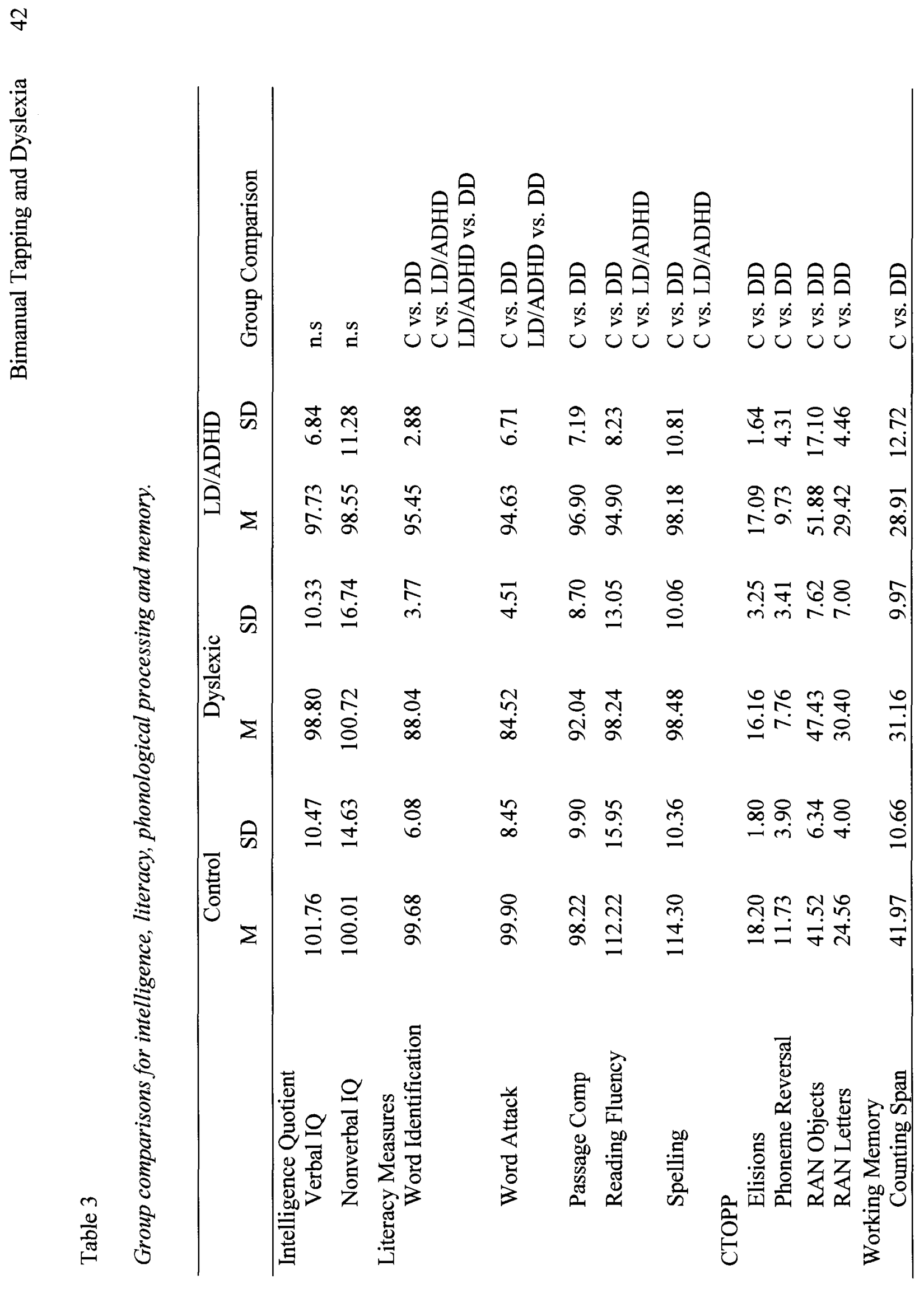
The omnibus tests revealed significant main effects for Word Identification, $F(2$, $35.56)=57.21, p<.001, \eta_{\mathrm{p}}^{2}=.48$, Word Attack, $F(2,27.78)=58.73, p<.001, \eta_{\mathrm{p}}^{2}=.45$, Passage Comprehension, $F(2,92)=3.86, p=.03, \eta_{\mathrm{p}}^{2}=.07$, Reading Fluency, $F(2,92)=$ $12.05, p=.001, \eta_{\mathrm{p}}^{2}=.21$, Spelling, $F(2,92)=26.53, p<.001, \eta_{\mathrm{p}}^{2}=.37$, Elisions, $F(2$, $28.60)=4.40, p=.022, \eta_{\mathrm{p}}^{2}=.14$, Phoneme Reversal, $F(2,94)=9.42, p<.001, \eta_{\mathrm{p}}^{2}=.17$, RAN Letters, $F(2,23.54)=9.34, p=.001, \eta_{\mathrm{p}}^{2}=.21$, RAN Objects, $F(2,21.84)=5.81, p=$ $.01, \eta_{\mathrm{p}}^{2}=.12$, and Counting Span, $F(2,94)=10.06, p<.001, \eta_{\mathrm{p}}^{2}=.18$. Only, Verbal IQ, $F(2,92)=1.23, p=.30$ and Nonverbal IQ, $F(2,92)=.08, p=.92$ failed to reach significance. To further analyze group differences, post-hoc analyses were used. Measures that were found to violate the assumption of homogeneity of variances were analyzed with the Games-Howell test (e.g., Word Identification, Word Attack, Elisions, RAN Objects and Letters), whereas the other measures were analyzed with the Sidak post-hoc test (i.e., Passage Comprehension, Reading Fluency, Spelling, Phoneme Reversal and Counting Span). The groups that were found to differ significantly, according to the post-hoc comparisons, are reported in Table 3.

As expected, the DD group obtained significantly lower scores on Word Identification and Word Attack tests (both $p s<.001$ ) as compared to the control participants. The mean differences for Word Identification and Word Attack test scores were 11.64 and 15.36, respectively. Hence, the selection criterion for dyslexia yielded a group of participants whose basic word recognition and decoding skills were significantly lower than that of control participants. In addition, participants in the DD group obtained significantly lower scores than the participants in the control group for all the remaining measures including Passage Comprehension $(p=.02)$, Reading Fluency $(p<.001)$, 
Spelling $(\mathrm{p}<.001)$, Elisions $(p=.016)$, Phoneme Reversal $(p=.001)$, RAN Letters $(p=$ $.001)$, RAN Objects $(p=.007)$ and Counting Span $(\mathrm{p}<.001)$

The comparisons between the $\mathrm{DD}$ and $\mathrm{LD} / \mathrm{ADHD}$ groups revealed only two differences. The $\mathrm{LD} / \mathrm{ADHD}$ group was significantly better at single word recognition as measured by the Word Identification test $(p<.001)$ and decoding as measured by the Word Attack test $(p=.001)$. All other differences were not significant.

Finally, the comparison between the LD/ADHD group and the Control group identified significant differences on Word Identification, Reading Fluency and Spelling. Thus, on the Word Identification test, the LD/ADHD group did not recognize as many real words as the control group $(p=.001)$ and were significantly slower on the test of Reading Fluency $(p=.001)$. The LD/ADHD groups' mean Spelling score was also significantly lower the Control group's score $(p<.001)$. No other differences were significant, but a trend was found for Counting Span $(p=.07)$ and RAN Letters $(p=.07)$. Bimanual Tapping Task Analyses

One of the main objectives of this study was to compare normal and dyslexic participants on the bimanual tapping task using the optimal task parameters suggested by Wolff et al. (1990). Tapping variability was the central variable to be examined. It was measured by the standard deviation of the Inter-Response-Interval (IRI). In addition, to gain a better understanding of the group performances under different task conditions, tapping average (i.e. ability to tap at the specified speed) was also analyzed. Finally, for the $1: 2$ ratio conditions, participants' ability to tap the prescribed ratio was evaluated.

The data in this section reflect performances measured for 30 seconds once the metronome had stopped (i.e., we measured unpaced tapping). The data obtained for the 
left and right fingers were analyzed separately to control for the number of expected tapping responses at both prescribed tapping speeds (Wolff et al., 1990). This was necessary because of the $1: 2$ ratio conditions, which required participants to tap at the prescribed speed with the right hand (e.g. $120 \mathrm{BPM}$ ) and at half the prescribed speed with the left hand (e.g. 60 BPM). Hence, the right finger in the 1:1 simultaneous condition was compared to the right finger of the $1: 2$ ratio condition and the left finger of the $1: 1$ alternation condition was compared to the left finger of the 1:2 ratio condition. Finally, age was used as a covariate in all tapping analyses to control for the age difference that was found between LD/ADHD and other participants. The descriptive statistics for tapping variability and tapping average obtained under all conditions are presented in Appendix A.

In the demographics section, it was found that the proportion of individuals who expressed a left hand preference was higher in the control group than in the two other groups. Considering that differences in brain lateralization have been found between leftand right-handed individuals (Banich, 2004) and that lateralization may have an impact on bimanual tapping, this analysis, as well as all the analyses presented in this section were conducted without the left-handed individuals $(n=82)$. The results of the analyses did not differ from those that included all individuals $(\mathrm{N}=95)$. Thus, the following analyses included all participants.

Tapping variability (IRI) for the Right hand. A (2 X 2) X 3 Mixed-Design ANCOVA with Speed (120 BPM and 184 BPM) and Task (1:1 simultaneous and 1:2 ratio) as the within-subjects factor and Group (control, DD, and LD/ADHD) as the between-subjects factor was conducted on the right hand tapping variability. The Speed 
$\mathrm{X}$ Task $\mathrm{X}$ Group interaction was not significant $F<1$, nor was the Speed $\mathrm{X}$ Group, $F$ (2, $91)=1.26$, or Speed $X$ Ratio interaction, $F(2,91)=1.09$. A Task X Group interaction was found, however, $F(2,91)=6.10, p=.003, \eta_{\mathrm{p}}^{2}=.12$. An examination of Figure 2 indicated that the tapping variability for all participants increased significantly at the $1: 2$ ratio. A simple effect analysis revealed that tapping variability did not differ between groups in the 1:1 simultaneous tapping condition, $F<1$, but did differ in the 1:2 ratio condition, $F(2,91)=5.36, p=.006, \eta_{\mathrm{p}}^{2}=.07=.11$. Sidak-adjusted post-hoc comparisons revealed that the Control group tapped with less variability than both the DD $(p=.03)$ and the LD/ADHD group $(p=.04)$. Main effects were found for Task, $F(1,91)=14.57, p$ $<.001, \eta_{\mathrm{p}}^{2}=.14$ and Group, $F(1,91)=14.57, p<.001, \eta_{\mathrm{p}}^{2}=.14$, but not for speed, $(\mathrm{F}=$ 2.73).

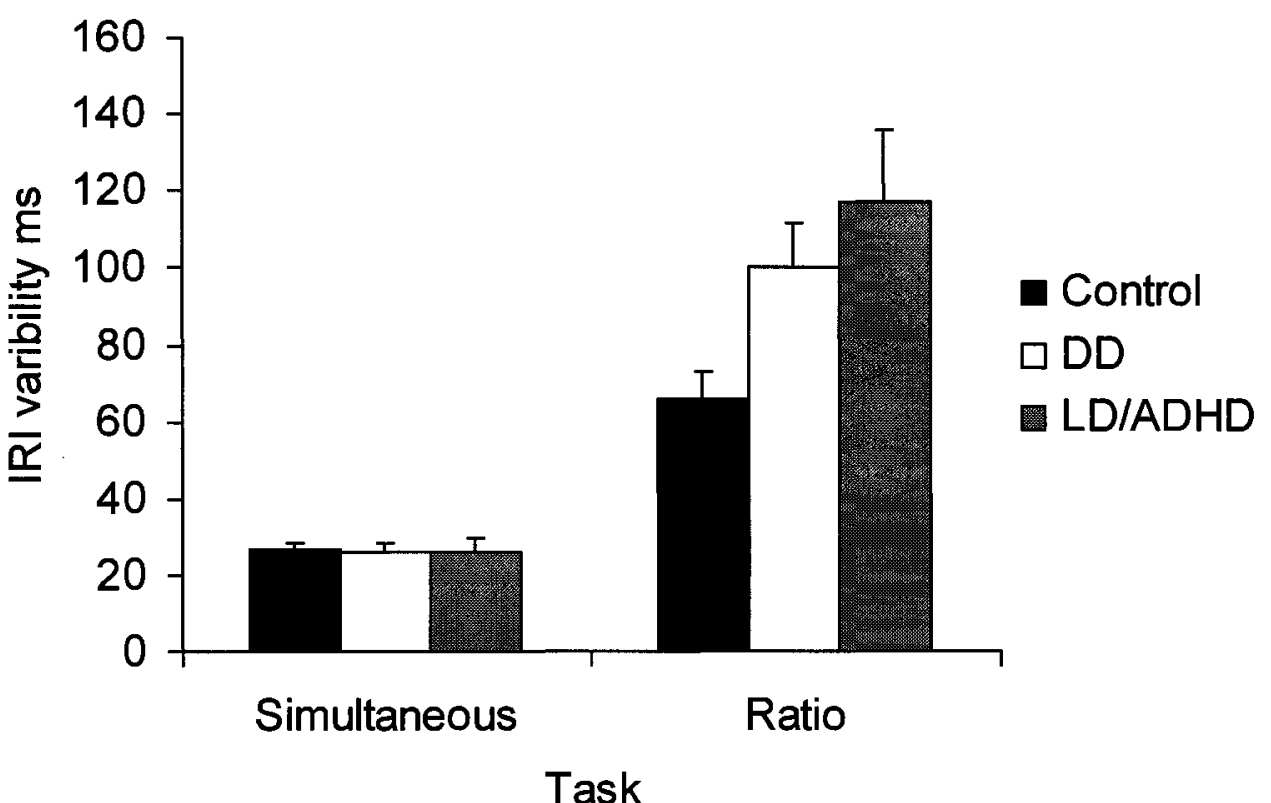

Figure 2. Right hand tapping variability (ms) by Task (1:1 simultaneous, 1:2 ratio) and Group (Control, DD, LD/ADHD) collapsed across Speed with (SE bars).

Tapping Average of the (IRI) for the Right hand. A (2 X 2) X 3 Mixed-Design ANCOVA with Speed (120 BPM and 184 BPM) and Task (simultaneous 1:1; and ratio 
$1: 2)$ as the within-subjects factor and Group (Control, DD, and LD/ADHD) as the between-subjects factor was conducted on the right hand tapping averages. There was a significant three-way interaction between Speed X Task X Group, $F(2,91)=4.401, p=$ $.015, \eta_{\mathrm{p}}^{2}=.09$, which is depicted in Figure 3 and Figure 4. The average time between each beat for $120 \mathrm{BPM}$ was expected to be $500 \mathrm{~ms}$ whereas for $184 \mathrm{BPM}$ the average time between each beat was expected to be $326 \mathrm{~ms}$. Thus, if participants had been able to comply perfectly with task demands, only a main effect of speed would have been found. Yet, Figure 3 presents a different pattern of results.

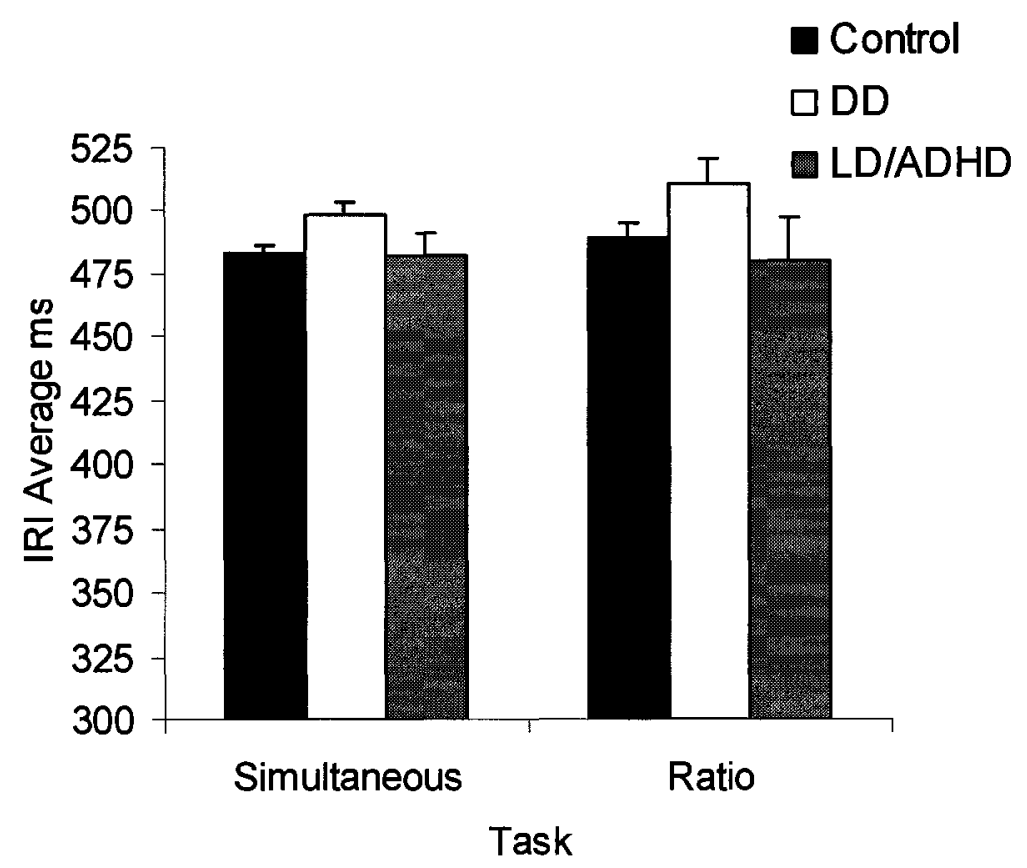

Figure 3. Right hand tapping average (ms) as a function of Task (1:1 simultaneous, $1: 2$ ratio) by Group (Control, DD, LD/ADHD) at the slow Speed (120 BPM) with (SE bars) 


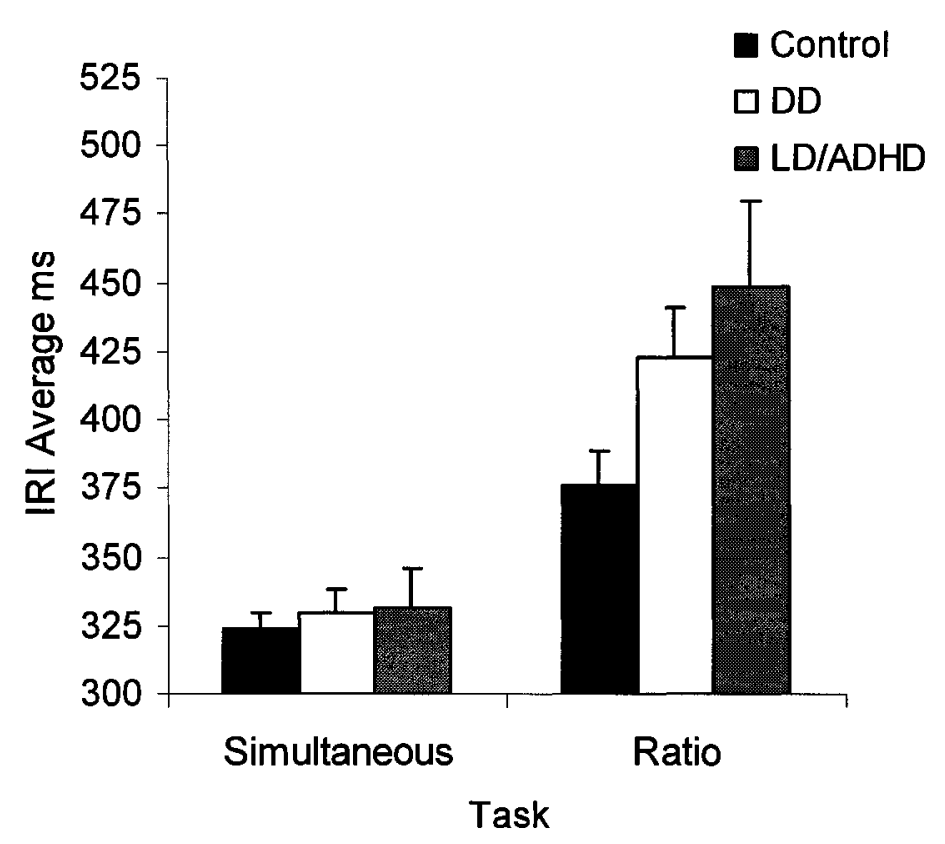

Figure 4. Right hand tapping average (ms) as a function of Task (1:1 simultaneous, $1: 2$ ratio) by Group (Control, DD, LD/ADHD) at the fast Speed (184 BPM) with (SE bars)

The three groups were on average tapping close to the expected $500 \mathrm{~ms}$ in the slow (120 BPM) condition for both simultaneous and ratio tapping patterns. Similarly, as seen in Figure 4 participants seem to have been able to maintain the expected rhythm in the fast (184 BPM) simultaneous condition, however, at the 1:2 ratio condition all participants appeared to have slowed down. To verify these impressions, the three-way interaction was decomposed into simple interaction effects of Task X Group at each level of Speed.

A significant effect was present for Task X Group at $184 \mathrm{BPM}, F(2,91)=3.34, p$ $=.04, \eta_{\mathrm{p}}^{2}=.07$, but not at $120 \mathrm{BPM}, F<1$. The 2-way interaction between Task and Group at 184 BPM was further decomposed into second-order simple effects. The tapping average did not differ by Group in the 1:1 simultaneous condition, $F<1$, but did differ in the $1: 2$ ratio condition, $F(2,91)=3.69, p=.029, \eta_{\mathrm{p}}^{2}=.08$. Sidak-adjusted post- 
hoc comparisons revealed that excluding the difference found between the control and LD/ADHD groups, no other difference was significant.

Tapping variability of the (IRI) for the Left-hand. A (2 X 2) X 3 Mixed-Design ANCOVA with Speed (120 BPM and 184 BPM) and Task (1:1 alternation; and 1:2 ratio) as the within-subjects factor and Group (control, DD, and LD/ADHD) as the between subjects factor was conducted on the left hand tapping variability. The Speed X Task X Group interaction was not significant, $F(2,91)=.77, p=.47, \eta_{\mathrm{p}}^{2}=.02$. There were, however, two significant two-way interactions.

First, the Task X Speed interaction was found to be significant, $F(1,91)=3.99, p$ $=.05, \eta_{\mathrm{p}}^{2}=.04$. The data are shown in Figure 5. Simple effect analyses revealed that tapping variability was greater in the $1: 2$ ratio condition than it was in the $1: 1$ alternation condition at both $120 \mathrm{BPM}, F(1,91)=25.22, p<.001, \eta_{\mathrm{p}}^{2}=.22$, and $184 \mathrm{BPM}, F(1,91)=$ 97.44, $p<.001, \eta_{\mathrm{p}}^{2}=.52$. Next, the effect of Speed at each level of task (alternation or ratio) was examined. Speed did not influence tapping variability at the 1:1 alternation task, $F<1$, but did significantly effect tapping variability at the ratio task, $F(1,91)=$ $19.06, p<.001, \eta_{\mathrm{p}}^{2}=.17$, indicating that tapping variability was significantly higher at $184 \operatorname{BPM}(M=162.99, S E=11.36)$ compared to $120 \mathrm{BPM}(M=48.19, S E=3.68)$ for all three groups. 


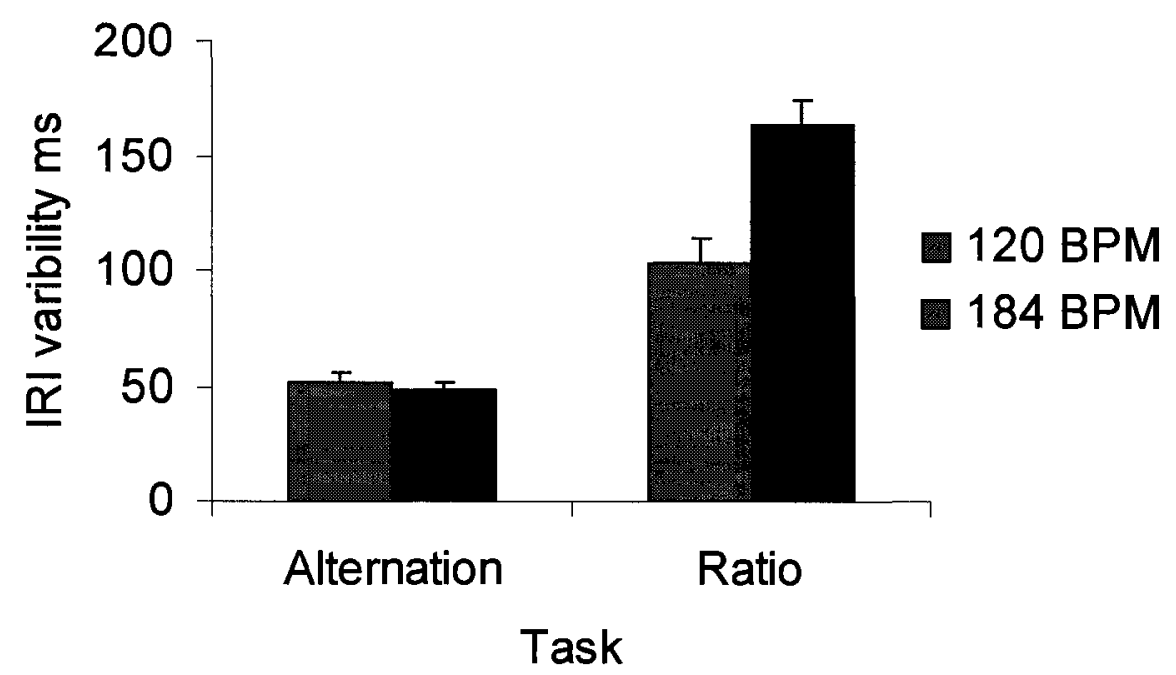

Figure 5. Left hand tapping variability $(\mathrm{ms})$ as a function of Task (1:1 alternation and $1: 2$ ratio) by Speed (120 BPM, 184 BPM) collapsed across Groups with (SE bars)

There was also a significant interaction effect of Task $\mathrm{x}$ Group, $F(2,91)=7.75, p$ $=.001, \eta_{\mathrm{p}}^{2}=.15$. As can be seen in Figure 6, tapping variability appears to differ between groups at the (1:2) ratio task. Simple effect analyses confirmed this impression. Groups were different at the $1: 2$ ratio condition, $F(2,91)=8.44, p<.001$, $\eta_{\mathrm{p}}^{2}=.16$, but not the (1:1) alternation condition, $F=1.81$. Sidak-adjusted post-hoc comparisons revealed the control group was significantly less variable than the DD group $(p=.001)$ and the LD/ADHD group $(p=.03)$. The latter two groups did not differ $(p=.99)$.

The main effect of Task was also significant, $F(1,91)=13.64, p<.000, \eta_{\mathrm{p}}^{2}=.13$, again indicating that $1: 2$ ratio created more variability than the $1: 1$ alternation task.

Finally, although there was a main effect of Speed, $F(1,91)=8.34, p=.005, \eta_{\mathrm{p}}^{2}=$ .08 , Speed did not interact with Group, $F(1,91)=8.34, p=.005, \eta_{\mathrm{p}}^{2}=.08$. This result suggest that although tapping a 1:2 ratio at 184 BPM was more difficult than tapping the 1:2 ratio at $120 \mathrm{BPM}$, no group had significantly more problems at the fast speed. 


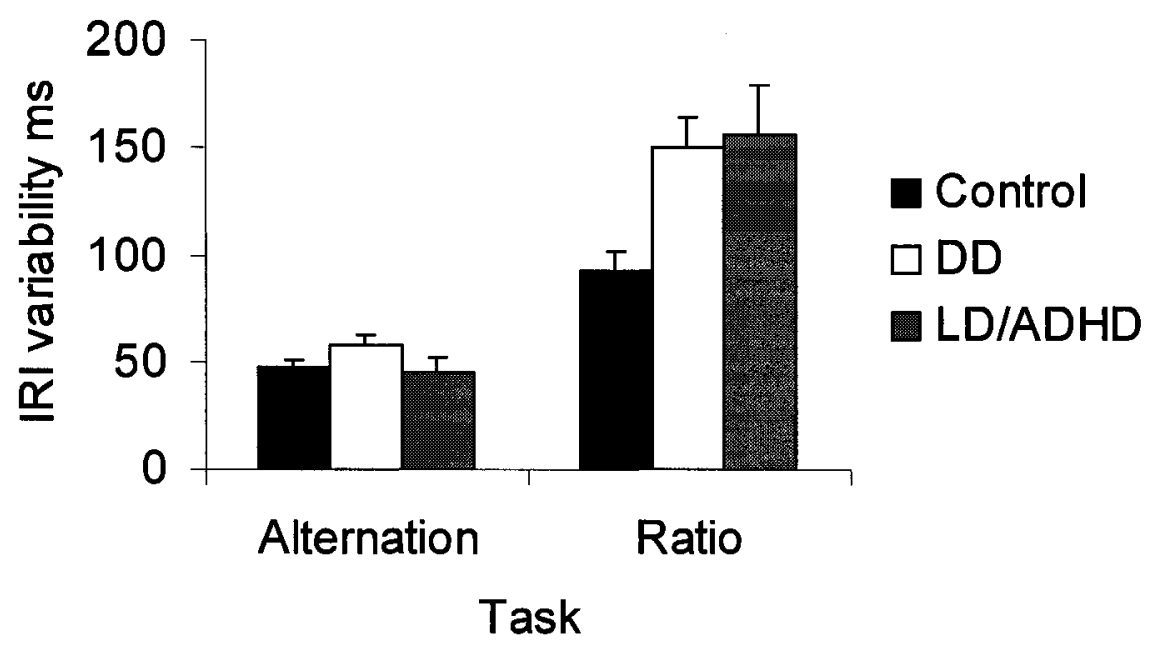

Figure 6. Left hand tapping variability (ms) as a function of Task (1:1 alternation, 1:2 ratio) by Group (Control, DD, LD/ADHD) collapsed across Speed with (SE bars) Average Tapping of the (IRI) for the Left hand. A (2 X 2) X 3 Mixed-Design ANCOVA with Speed (120 BPM and 184 BPM) and Task (1:1 alteration and 1:2 ratio) as the within-subjects factor and Group (control, DD, and LD/ADHD) as the between subjects factor was conducted on the left hand tapping average. The three-way interaction was marginally significant, $F(2,91)=2.72, p=.07, \eta_{\mathrm{p}}^{2}=.06$ (see Figure 7 and Figure 8). 


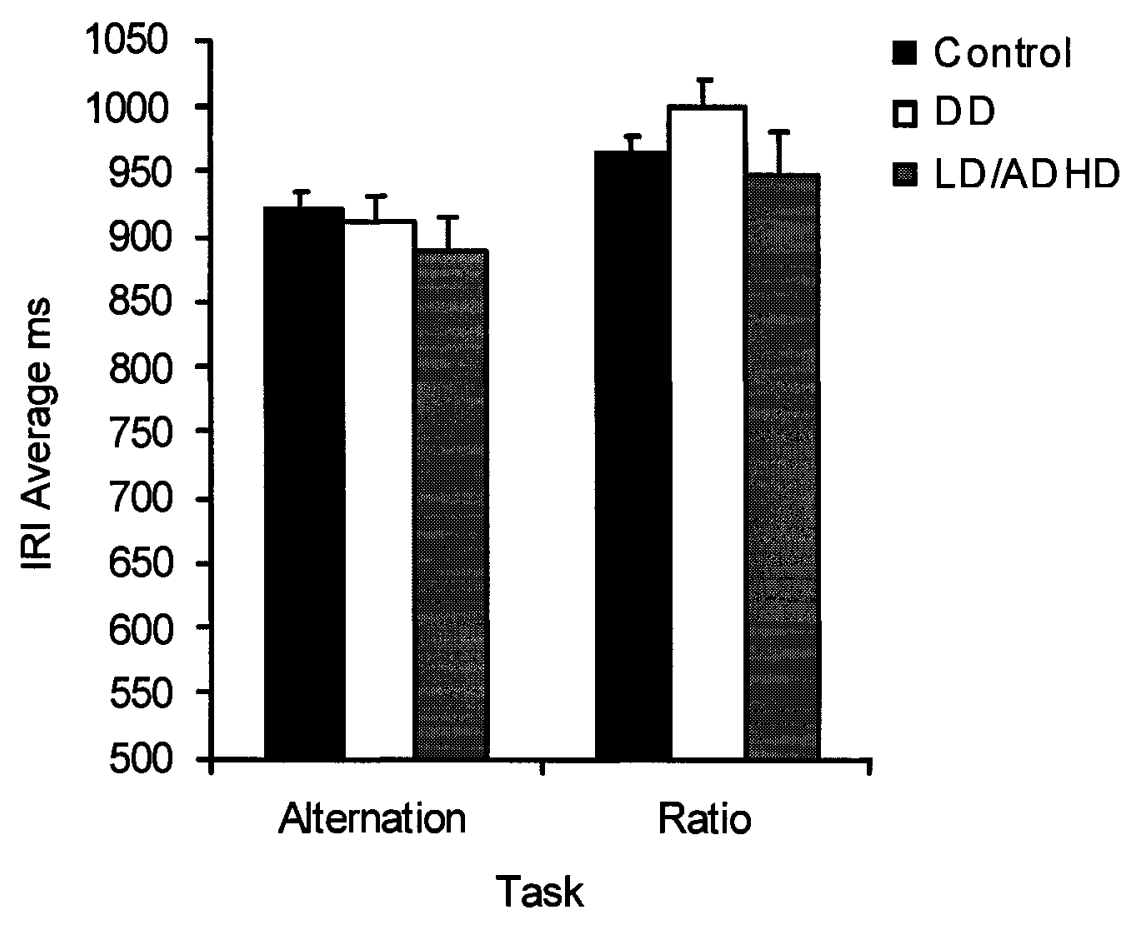

Figure 7. Left hand tapping average (ms) as a function of Task (1:1 alternation, 1:2 ratio) by Group (control, DD, LD/ADHD) at the slow Speed (120 BPM) with (SE bars)

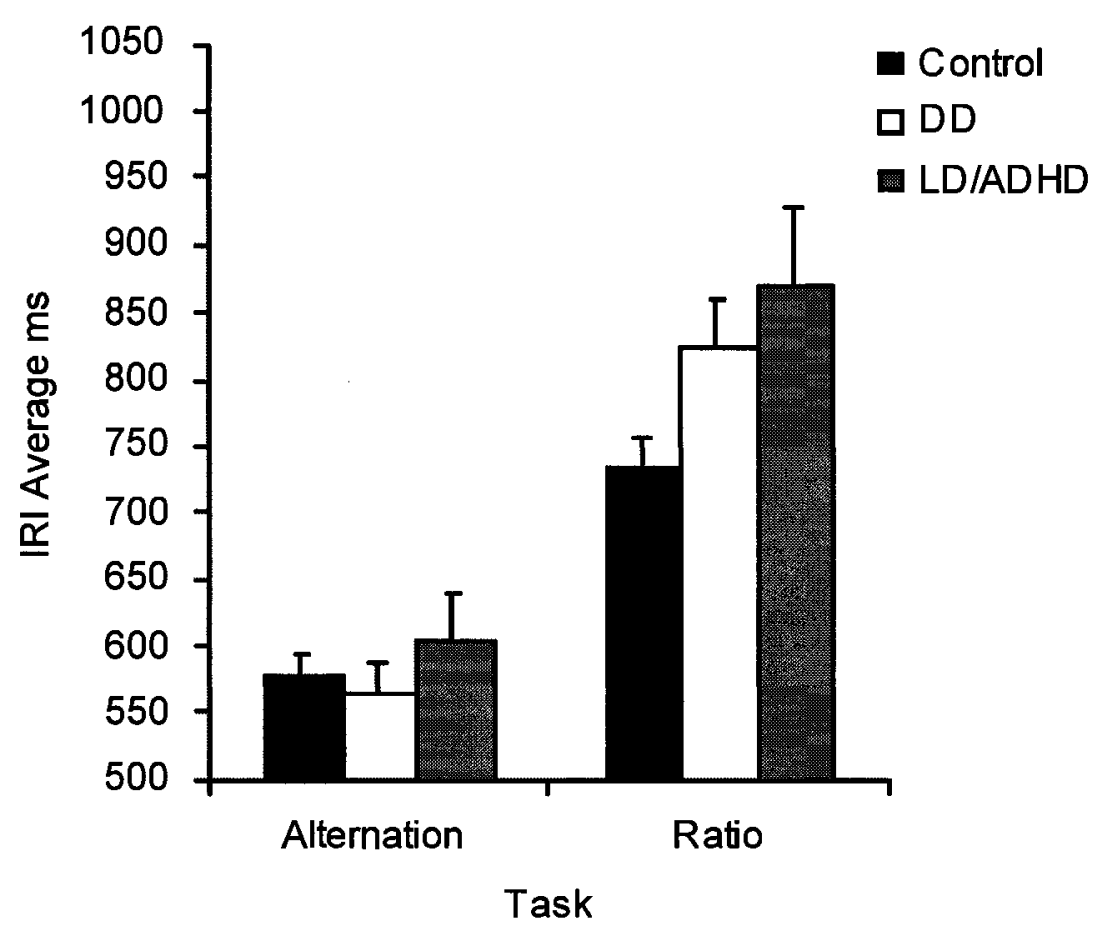

Figure 8. Left hand tapping average (ms) as a function of Task (1:1 alternation, $1: 2$ ratio) by Group (Control, DD, LD/ADHD) at the fast Speed (184 BPM) with (SE bars) 
Once again, if participants had complied perfectly with task demands, only a main effect of speed should have been found. Nevertheless, the pattern of results is similar to that found for the right hand tapping average. Participants in all groups showed similar performances in all conditions except in the 184 BPM 1:2 ratio condition. Considering that the Task X Group effect at the fast speed would yield the most theoretically relevant results, simple interaction effects were analysed even though the three-way interaction did not quite reach significance. We note, however, that the Speed X Group interaction, $F(2,91)=3.90, p=.024, \eta_{\mathrm{p}}^{2}=.14$, the Task X Group interaction, $F(2,91)=3.74, p=.03$, $\eta_{\mathrm{p}}^{2}=.08$, and the main effect of Speed, $F(2,91)=15.51, p<.001, \eta_{\mathrm{p}}^{2}=.15$, were significant.

As could be expected from Figure 8, a significant effect was present for Task $\mathrm{X}$ Group at $184 \mathrm{BPM}, F(2,91)=4.17, p=.02, \eta_{\mathrm{p}}^{2}=.08$, but not at $120 \mathrm{BPM}, F(2,91)=$ $1.69, p=.19, \eta_{\mathrm{p}}^{2}=.04$ (see Figure 7). The 2-way simple interaction between Task and Group at 184 BPM was further decomposed into second-order simple effects. The tapping average did not differ by Group in the $1: 1$ alternation condition, $F(2,91)=.46, p$ $=.63, \eta_{\mathrm{p}}^{2}=.01$, but did differ in the $1: 2$ ratio condition, $F(2,91)=4.09, p=.02, \eta_{\mathrm{p}}^{2}=.08$. Sidak-adjusted post-hoc comparisons revealed that no other difference in tapping average other than that between the control group and the LD/ADHD group was significant ( $p$ s > $.14)$.

\section{Tapping Pattern}

The tapping pattern for the 1:2 ratio condition was the number of expected tapping responses by the right finger divided by the number of expected tapping responses by the left finger. Thus, participants should obtain a tapping ratio of 2 if they 
comply perfectly with task demands. Following Wolff et al. (1990), tapping ratios between 1.8 and 2.2 were considered to be within an acceptable range (i.e. participants maintained the prescribed ratio). Ratios outside of that range were considered deviant. At 120 BPM, 57 control participants (97\%), 24 dyslexic participants (96\%) and the 11 LD/ADHD participants (100\%) were within normal range. Hence, at that speed, the majority of participants in all groups were able to tap at the prescribed ratio. At 184 BPM, however, the results were different. Whereas 55 control participants $(93 \%)$ and the $11 \mathrm{LD} / \mathrm{ADHD}$ participants $(100 \%)$ were within the acceptable range, only 20 dyslexic participants $(75 \%)$ tapped the prescribed $(1: 2)$ ratio. Thus, $25 \%$ of dyslexic participants tapped at a deviant ratio.

\section{Correlations among the Variables}

Before investigating the relation between bimanual tapping and reading ability, correlations among reading, intelligence, spelling, phonological processing, rapid naming and working memory measures were first computed to ensure that the sample exhibited a pattern of performance in line with previous research. Age-normed Standardized Scores for the WJ-III reading tests, WRAT-3 spelling test; and KBIT-2; and the standardized residual scores (with age partialled out) for Elisions, Phoning Reversal, RAN Objects and Letters, and Counting Span were entered in the correlation matrix. Residual scores were used for the five latter tests because the age norms for the CTOPP (Elisions, Phoning Reversal, RAN Objects and Letters) did not cover the sample's age range and the Counting Span test did not have age norms. The correlations are shown in Table 4. 


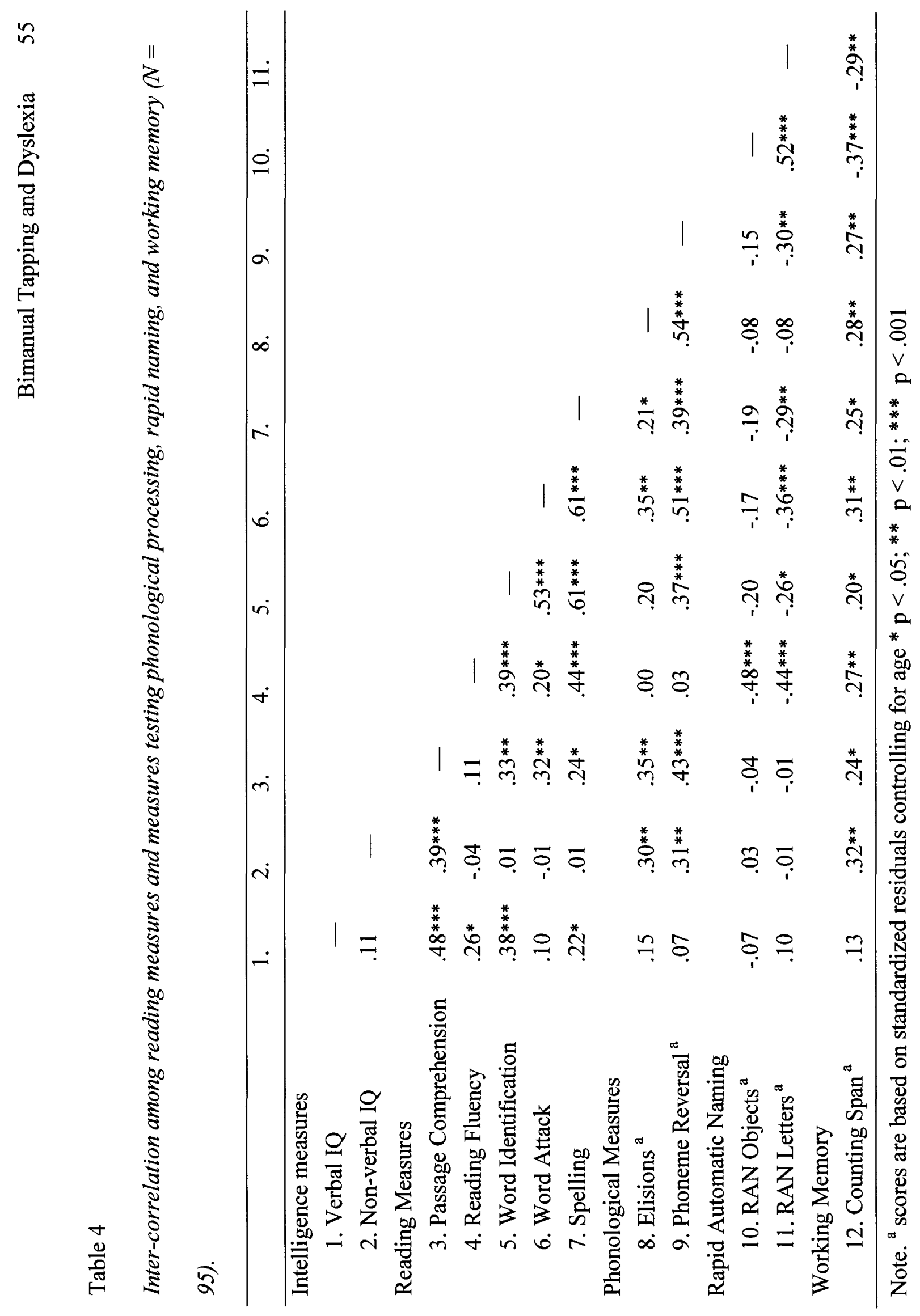


As expected, most reading measures showed medium to large correlations among them. The only two exceptions were the correlations between Word Attack and Reading Fluency $(r=.20)$, and between Reading Fluency and Passage Comprehension $(r=.11)$. Nevertheless, these correlations are similar to those that have been found in previous research (Benson, 2008; Chiappe et al., 2002; Swanson, Trainin, Necoechea, \& Hammill, 2003).

In addition, there was a clear relationship between the two measures of phonological processing $(r=.54)$ and predictably, both Elisions and Phoneme Reversal were correlated with Word Attack $(r=.35$ and $r=.51)$. However, the correlations between the literacy measures and Phoneme Reversal were stronger than those between the literacy measures and Elisions. Considering that the scores for the Elision test were close to ceiling (See Table 2), it is probable that this task was too easy for most participants. Hence, the Elision test's ability to be strongly related with the other variables was reduced.

The two measures of Rapid Automatic Naming were also clearly related $(r=.52)$. Both RAN Object and RAN Letters were strongly correlated with Reading Fluency $(r=-$ .48 and $r=-.44$, respectively), and RAN Letters showed small to medium correlations with all other measures of literacy except Passage Comprehension $(r=-.01)$. These results are also in line with those found in previous research with adults (see Swanson et al., 2003).

The IQ measures were not significantly correlated with each other $(r=.11)$. The Verbal IQ score was strongly correlated with Word Identification and Passage Comprehension ( $r=.38$ and $r=.48$, respectively). It also displayed a small correlation 
with Reading Fluency $(r=.26)$ and Spelling $(r=.22)$, but it was not significantly correlated with any other variable. The Non-Verbal IQ score was correlated with Passage Comprehension, Counting Span, and both phonological measures ( $r$ s between .30 and .39), but was not correlated with any other measure. Finally, Counting Span showed significant, but mostly small correlations with all the variables except Verbal IQ $(r=.13)$. Table 5 presents the next set of correlations examining the relationship between tapping variability (in the 184 BPM 1:2 ratio condition), and reading, spelling, rapid naming, intelligence, and working memory.

The correlations show that tapping variability in the left hand was associated with decreased performance on the majority of measures. Left hand tapping variability was significantly correlated with all the measures of literacy and the correlations were strongest with Word Identification $(r=-.38)$ and Spelling $(r=-.35)$. However, left hand tapping variability was also correlated with predictors of literacy, namely phonological processing and rapid naming, and Counting Span. Thus, it is impossible to ascertain from these correlations if tapping variability can explain unique variance in any of the literacy measures that could not be otherwise explained by appealing to the established predictors of reading ability such as phonological processing, working memory or RAN. Right hand tapping variability was significantly correlated with Reading Fluency and Spelling, but also Phoneme Reversal, both RAN tasks and Counting Span.

\section{Stepwise Regressions}

To determine if tapping variability could contribute uniquely to different aspects of literacy, five separate stepwise regression analyses were conducted with Word Attack, 
Word Identification, Spelling Reading Fluency, and Passage Comprehension serving in turn as the criterion variable.

\section{Table 5}

Correlation between Literacy variables and tapping variability for the 1:2 ratio at 184 $B P M$ for the left and right fingers.

\begin{tabular}{lll}
\hline & \multicolumn{2}{c}{ IRI variability } \\
\cline { 2 - 3 } & \multicolumn{1}{c}{ Left } & Right \\
\hline Literacy Variables & $-.38^{* * *}$ & -.16 \\
Word Identification & $-.22^{*}$ & -.17 \\
Word Attack & $-.20^{*}$ & -.16 \\
Passage Comprehension & $-.30^{* *}$ & $-.18^{*}$ \\
Reading Fluency & $-.35^{* * *}$ & $-.21^{*}$ \\
Spelling & & \\
Intelligence measure & -.16 & -.09 \\
Verbal IQ & .00 & -.13 \\
Non-Verbal IQ & & \\
Phonological measures & $-.29^{* *}$ & $-.24^{* *}$ \\
Phoneme Reversal & -.04 & -.04 \\
Elisions & & \\
Rapid Automatic Naming & $.29^{* *}$ & $.30^{* *}$ \\
RAN Objects & $.23^{*}$ & $.21^{*}$ \\
RAN Letters & & \\
Working memory & $-.28^{* *}$ & $-.25^{* *}$ \\
Counting Span & & \\
\hline
\end{tabular}

${ }^{*} p<.05 ;{ }^{* *} p<.01 ;{ }^{* *} p<.001,1$-tailed test

For each stepwise regression, the predictor variables were selected as follows. First, any intelligence, phonological processing, rapid naming, or the working memory measure that displayed a zero-order correlation with the criterion (as shown in Table 4) 
was included in the regression. Then, if the right or left finger tapping variability measure displayed a zero-order correlation with the criterion (as shown in Table 5), it was also included in the regression. Note that the residual tapping scores (with age partialled out) were used. Finally, the entry criteria for the predictor variables in the stepwise regressions were set at $F \leq .05$ and the removal criteria were set at $F \geq .10$.

The first stepwise regression included Word Attack as the criterion and Phoneme Reversal, Counting Span, RAN Letters, Elisions and left hand IRI variability (L-IRI variability) as the predictors. The results of the regression are shown in Table 6. It can be observed that Phoneme Reversal and RAN Letters explained $31 \%$ of the variance and that L-IRI variability accounted for no variance once the former two variables had been entered in the regression. Hence, left hand tapping variability does not appear to explain any unique variance in the participants' Word Attack scores once measures of phonological processing and rapid naming have been taken into account.

On the other hand, taking into consideration that Word Attack and Phoneme Reversal are both decoding measures, it could be argued that the latter variable unduly biases the regression against finding that L-IRI explains unique variance in the criterion variable. Although this possibility is unlikely considering that RAN Letter did explain a significant amount of variance once Phoneme Reversal had been included in the regression, a second regression involving Word Attack as the criterion was conducted. The predictors were Counting Span, RAN Letters and L-IRI. RAN Letters and Counting Span accounted for $17 \%$ of the variance, $F(2,94)=9.70, p<.001$, but L-IRI once again failed to explain any unique variance. Thus, it can be safely concluded that tapping variability is not related to phonological processing ability. In view of this result, I 
decided to use Word Attack as the measure of decoding ability in the remaining regression analyses to make the results more comparable to previous studies (e.g. Chiappe et al., 2002).

Table 6

Stepwise regression analysis predicting adults' WJ-III Word Attack performance.

\begin{tabular}{lrccccccc}
\hline $\begin{array}{c}\text { Step and } \\
\text { Variable }\end{array}$ & $\mathrm{b}$ & $S E \mathrm{~b}$ & $\beta$ & $t$ & $R^{2}$ & $\Delta R^{2}$ & $\begin{array}{c}\text { Overall } \\
F\end{array}$ & $\begin{array}{c}\text { Partial } \\
r^{2}\end{array}$ \\
\hline Step 1 & & & & & & & $F$ & \\
$\quad$ Phoneme Rev. & 5.09 & 0.89 & 0.51 & $5.73^{* * *}$ & .26 & .26 & $32.77^{* * *}$ & .26 \\
Step 2 & & & & & & & & \\
$\quad$ Phoneme Rev. & 4.42 & 0.91 & 0.44 & $4.88^{* * *}$ & & & & .20 \\
RAN Letters & -2.22 & 0.91 & -0.22 & $-2.45^{*}$ & .31 & .05 & $20.25^{* * *}$ & .06 \\
Excluded Var. & & & & & & & & \\
Counting Sp & & & 0.15 & 0.12 & & & & .03 \\
Elisions & & & 0.13 & 0.23 & & & & .02 \\
L-variability & & & -0.04 & 0.67 & & & & .00 \\
\hline
\end{tabular}

Note: $t$, testing the beta weights linear relationship ${ }^{*} p<.05 ;{ }^{* * *} p<.001$; to Word Attack.

The next measure of literacy examined was Word Identification. Five predictor variables were entered into the model: Word Attack, Verbal IQ, L-IRI, Counting Span and RAN Letters. As shown in Table 7, Word Attack, Verbal IQ, and L-IRI variability accounted for approximately $44.1 \%$ of the variance in Word Identification, $F(3,91)=$ $23.92, p<.001$. Word Attack entered first accounting for $28 \%$ of variance, Verbal IQ entered second explaining an additional $10.7 \%$ of variance, and L-IRI entered third explaining an additional 5.3\%. Thus, this regression analysis suggests that tapping variability can explain unique variance in a measure of reading ability even when other 
plausible explanatory factors are allowed to compete for variance (e.g. IQ and working memory) and that a measure of phonological processing has already been entered into the equation.

Table 7

Stepwise regression analysis predicting adults' WJ-III Word Identification performance.

\begin{tabular}{ccccccccc}
\hline $\begin{array}{c}\text { Step and } \\
\text { Variable }\end{array}$ & $\mathrm{b}$ & $S E \mathrm{~b}$ & $\beta$ & $t$ & $R^{2}$ & $\Delta R^{2}$ & $\begin{array}{c}\text { Overall } \\
F\end{array}$ & $\begin{array}{c}\text { Partial } \\
r^{2}\end{array}$ \\
\hline Step 1 & & & & & & & & \\
Word Attack & 0.39 & 0.06 & 0.53 & $6.02^{* * *}$ & .28 & .28 & $36.21^{* * *}$ & .28 \\
Step 2 & & & & & & & & \\
Word Attack & 0.36 & 0.06 & 0.50 & $6.06^{* * *}$ & & & & .29 \\
Verbal I.Q & 0.24 & 0.06 & 0.33 & $4.02^{* * *}$ & .39 & .11 & $29.13^{* * *}$ & .15 \\
Step 3 & & & & & & & & \\
Word Attack & 0.33 & 0.06 & 0.45 & $5.57^{* * *}$ & & & & .25 \\
Verbal IQ & 0.21 & 0.06 & 0.30 & $3.71^{* * *}$ & & & & .13 \\
L-variability & -1.74 & 0.59 & -0.24 & $-2.94^{* *}$ & .44 & .05 & $23.92^{* * *}$ & .09 \\
Excluded Var. & & & & & & & & \\
Counting Sp. & & & -0.05 & -0.56 & & & & .01 \\
RAN Letters & & & -0.09 & -1.02 & & & & .00 \\
\hline
\end{tabular}

Note: $t$, testing the beta weights linear relationship ${ }^{* *} p<.01$; ${ }^{* * *} p<.001$; to Word Identification.

The third literacy measure examined was Spelling. This stepwise regression included six predictor variables: Word attack, left hand IRI variability (L-IRI variability), RAN Letters, right-hand IRI variability (R-IRI variability), counting span and Verbal IQ The overall model included two significant predictors of Spelling: Word Attack and LIRI. The two variables accounted for approximately $42.4 \%$ of variance. As shown in 
Table 8, Word Attack entered the model first explaining approximately $37.4 \%$ of the variance. $L-I R I$ variability explained an additional $5 \%$ of the variance.

Table 8

Stepwise regression analysis predicting adults' WRAT Spelling performance.

\begin{tabular}{ccccccccc}
\hline Step and Variable & $\mathrm{b}$ & $S E \mathrm{~b}$ & $\beta$ & $t$ & $R^{2}$ & $\Delta \mathrm{R}^{2}$ & $\begin{array}{c}\text { Overall } \\
\text { Partial }\end{array}$ \\
& & & & & & & $F$ & $r^{2}$
\end{tabular}

Step 1

$\begin{array}{lllllllll}\text { Word Attack } & 0.79 & 0.11 & 0.61 & 7.45^{* * *} & .37 & .37 & 55.49^{* * *} & .37\end{array}$

Step 2

$\begin{array}{llllllllll}\text { Word Attack } & 0.73 & 0.11 & 0.56 & 6.93^{* * *} & & & & .34 \\ \text { L - variability } & -2.96 & 1.05 & -0.23 & -2.83^{* *} & .42 & .05 & 33.83^{* * *} & .08\end{array}$

Excluded Var.

$\begin{array}{lll}\text { RAN Letters } & -0.05 & -0.55\end{array}$

$\begin{array}{llll}\text { R- variability } & 0.15 & 1.23 & .02\end{array}$

$\begin{array}{llll}\text { Counting Sp } & 0.02 & 0.18 & .00\end{array}$

$\begin{array}{llll}\text { Verbal IQ } & 0.13 & 1.63 & .03\end{array}$

Note: $t$, testing the beta weights linear relationship ${ }^{* *} p<.01 ;{ }^{* *} p<.001$; to Spelling.

A fourth Stepwise regression was conducted with Reading Fluency as the criterion variable. The predictors were RAN Objects, RAN Letters, Verbal IQ, L-IRI, Counting Span, Word Attack, and R-IRI. The results of the regression are shown in Table 9. The overall model explained $35 \%$ of variance in Reading Fluency. RAN Objects, Verbal IQ, RAN Letters uniquely explained $23 \%, 5 \%$, and $7 \%$ of the variance respectively. Neither tapping variability measure explained a significant amount of variance. 
Table 9

Stepwise regression analysis predicting adults' WJ-III Reading Fluency performance

\begin{tabular}{ccccccccc}
\hline Step and Variable & $\mathrm{b}$ & $S E \mathrm{~b}$ & $\beta$ & $t$ & $R^{2}$ & $\Delta R^{2}$ & $\begin{array}{c}\text { Overall } \\
F\end{array}$ & $\begin{array}{c}\text { Partial } \\
r^{2}\end{array}$ \\
\hline Step 1 & & & & & & & & \\
RAN Objects & -7.74 & 1.49 & -0.48 & $-5.21^{* * *}$ & .23 & .23 & $27.17^{* * *}$ & .23 \\
Step 2 & & & & & & & & \\
RAN Objects & -7.47 & 1.45 & -0.46 & $-5.17^{* * *}$ & & & & .22 \\
Verbal IQ & 0.37 & 0.14 & 0.23 & $2.58^{* *}$ & .28 & .05 & $17.75^{* * *}$ & .07 \\
Step 3 & & & & & & & & .08 \\
RAN Objects & -4.70 & 1.63 & -0.29 & $-2.89^{* *}$ & & & & .10 \\
Verbal IQ & 0.44 & 0.14 & 0.28 & $3.21^{* *}$ & & & & .10 \\
RAN Letters & -5.22 & 1.63 & -0.32 & $-3.20^{* *}$ & .35 & .07 & $16.45^{* * *}$ & \\
Excluded Var. & & & & & & & & .02 \\
L-variability & & & -0.11 & -1.22 & & & & .00 \\
Counting Sp. & & & 0.04 & 0.46 & & & & .00 \\
Word Attack & & & 0.02 & 0.16 & & & & \\
R- variability & & & -0.00 & -0.01 & & & & \\
\hline
\end{tabular}

Note: $t$, testing the beta weights linear relationship ${ }^{* *} p<.01 ; * * * p<.001$ to Reading Fluency.

Passage Comprehension was the final reading measure examined. Five predictor variables were entered into the model: Verbal IQ, Non-Verbal IQ, Word Attack, Counting Span and Left-IRI. The results are shown in Table 10. Although Verbal IQ, Non-verbal IQ, and Word Attack explained approximately $42 \%$ of the variance, Left-IRI did not explain a sufficient amount of variance to be included in the final regression model. 
Table 10

Stepwise regression analysis predicting adults' WJ-III Passage Comprehension performance.

\begin{tabular}{lllllllll}
\hline Step and Variable & $\mathrm{b}$ & $S E \mathrm{~b}$ & $\beta$ & $t$ & $R^{2}$ & $\Delta R^{2}$ & Overall $F$ & $\begin{array}{c}\text { Partial } \\
r^{2}\end{array}$
\end{tabular}

Step 1

$\begin{array}{lllllllll}\text { Verbal IQ } & 0.46 & 0.09 & 0.48 & 5.30 * * * & .23 & .23 & 28.03 * * * & .23\end{array}$

Step 2

$\begin{array}{lllllllll}\text { Verbal IQ } & 0.42 & 0.08 & 0.45 & 5.24 * * * & & & & .23 \\ \text { Non-verbal IQ } & 0.22 & 0.06 & 0.34 & 3.98^{* * *} & .34 & .11 & 24.16^{* * *} & .15\end{array}$

Step 3

$\begin{array}{lllllllll}\text { Verbal IQ } & 0.40 & 0.08 & 0.42 & 5.16^{* * *} & & & .23 \\ \text { Non-verbal IQ } & 0.23 & 0.05 & 0.35 & 4.30^{* * *} & & & & .17 \\ \text { Word Attack } & 0.27 & 0.08 & 0.28 & 3.50^{* * *} & .42 & .08 & 22.15 * * * & .12\end{array}$

Excluded Var.

$\begin{array}{llll}\text { Counting Sp. } & -0.01 & -0.09 & .00\end{array}$

$\begin{array}{llll}\text { L-variability } & -0.08 & -0.91 & .01\end{array}$

Note: $t$, testing the beta weights linear relationship ${ }^{* * *} p<.001$ to Passage

Comprehension

\section{Discussion}

The first goal of the study was to determine if the Bimanual Tapping Task (BTT) differentiated between people with and without dyslexia as demonstrated by Wolff et al. (1990). Participants with dyslexia were expected to tap with greater variability at the fast (184 BPM) 1:2 ratio condition compared to controls. Moreover, the participants with dyslexia were not expected to differ compared to controls on the easier tapping patterns 
(1:1 symmetrical and 1:1 alternation). The results supported both hypotheses. These results thus replicate Wolff et al. (1990) and reveal that individuals with dyslexia tapped with more variability when the right and left fingers responded to different frequencies. The finding did not appear to be specific to dyslexia in the present sample, however, because greater tapping variability was also evident in the LD/ADHD group.

The second goal was to examine whether tapping variability explained unique variance in reading related skills. Stepwise regressions demonstrated that increased tapping variability in the left hand explained unique variance in Word Identification and Spelling, after phonological skills had been controlled for. Tapping variability did not explain unique variance in phonological processing, reading rate or comprehension. Thus, the pattern of results suggests that tapping variability may be related to impairments in the lexical rather than the non-lexical route to reading (Coltheart, Curtis, Atkins, \& Haller, 1993). The lexical route of reading is hypothesized to involve the visual representation o words in comparison to the non-lexical route, which involves the conversion of graphemes into phonemes (Weems \& Zaidel, 2004).

This theory may also help explain why increased tapping variability was not specific to dyslexia, but seemed to be more generally related to learning disabilities. In examining the differences and similarities among the groups in Table 3, it can be noted that the LD/ADHD group did not differ from controls on the phonological tasks, whereas the dyslexic group was significantly worse on all three phonological tasks. In contrast, both the LD/ADHD and dyslexic group were significantly impaired on Word identification and spelling compared to controls. Therefore, tapping variability may 
actually be picking up impairments in the lexical route to reading rather than the phonological route.

The discussion below will begin with a in-depth discussion about the bimanual tapping task, the relationship between tapping variability and reading related skills, limitations, and conclusions.

The Bimanual Tapping Task

Some authors have argued that individuals with dyslexia have difficulty processing temporal events that are presented at a fast rate. In a review paper, Wolff (1993) suggested that increasing the rate of tapping would result in a group by rate interaction showing that individuals with dyslexia would tap with greater variability at faster speeds. Wolff's (1993) prediction of a speed by group interaction was not found in this thesis, nor has it been found in other comprehensive studies (e.g., Chiappe et al., 2002; Ramus et al., 2003; Wolff et al., 1990).

However, speed does appear to be an important methodological task consideration because of the speed by task interaction (for the left hand). Specifically, tapping variability increased significantly at the (1:2) ratio at 184 BPM for all groups and this was not specific to dyslexia or LD/ADHD. Examination of the tapping pattern did indicate that participants were able to maintain the (1:2) ratio although they became more variable in their tapping. This finding is not surprising given the work of Repp (2003) who established that $240 \mathrm{BPM}$ was the approximate upper temporal threshold at which people could process temporal events. Outside this range, the BTT no longer assesses temporal processing (Repp, 2003). 
Speed as an important methodological consideration may be the reason that Ramus et al. (2003) did not find group differences on the $1: 2$ ratio task at the speed of $4 \mathrm{~Hz}(250 \mathrm{~ms}, 240 \mathrm{BPM})$. Examination of supplementary material indicated that the tapping variability in both groups was quite low (Control; $M=42.1 ; S D=23.4$ ) and (DD; $M=44.7 ; S D=21.2$ ) (see Ramus et al., 2003). The mean IRI variability for the $1: 2$ task in Ramus et al.'s study is very similar to our study's easier 1:1 tasks (see Appendix A). Ramus et al. did not report whether participants maintained the correct ratio. Therefore, it is possible that the required speed of tapping was outside participants' temporal thresholds, which consequently suggests that Ramus et al.'s task may not have reliably measured participants' ability to tap a 1:2 ratio. Thus, there is cause to question the validity of Ramus et al.'s conclusions regarding the relation between the BTT and reading.

The overall tapping averages for the $1: 2$ ratio indicated that maintaining a constant interval between beats was more difficult when the speed was 184 BPM than $120 \mathrm{BPM}$. The results for the right finger in the $184 \mathrm{BPM} 1: 2$ ratio condition revealed that all individuals tapped slower than the expected interval of $326 \mathrm{~ms}$. However, the DD group and LD/ADHD group were significantly slower than the control group. These results suggest that the participants in the DD and LD/ADHD groups both had more difficulty maintaining the ratio at $184 \mathrm{BPM}$ and slowed down in order to perform the task accurately. The tapping average for the left finger in the ratio condition also revealed that groups tapped slower than the expected interval of $652 \mathrm{~ms}$, and only the Control group differed from the LD/ADHD group. There was no difference evident between the tapping averages for the groups when performing the easier tapping task (i.e., 1:1 alternation) and 
this is similar to results reported by Chiappe et al. (2002). For both the right and left hands, the tapping average for 120 BPM did not produce group or task differences.

Finally, a deviant tapping pattern analysis indicated that at the slow speed the majority of participants were able to maintain the correct 1:2 ratio. Deviant patterns were more evident at the fast speed. Seven percent of controls, twenty-five percent of dyslexics and no LD/ADHD participants had difficulty maintaining the correct ratio at 184 BPM. Wolff et al. (1990) reported that approximately $46 \%$ of dyslexics had difficulty maintaining a (1:2) ratio compared to four percent in the control group.

In summary, participants with dyslexia tapped with greater variability at the $(1: 2)$ ratio at both 120 and $184 \mathrm{BPM}$. Thus, ratio was the most effective task manipulation to differentiate among the groups. Speed did increase the difficulty of the task in the left hand and this was specifically at the $1: 2$ ratio. The groups did not differ at the easier $(1: 1)$ tapping tasks and the importance of this finding will be discussed below. Finally, the finding did not appear to be specific to dyslexia because the LD/ADHD also tapped with more variability at the $(1: 2)$ ratio.

\section{The Relationship Between Tapping and Reading}

The correlations between tapping variability, literacy variables and cognitive variables replicate the results of previous research. For example, Swanson et al. (2003) conducted a meta-analysis that focused on the correlates of sight word reading. They concluded that the strongest correlates of word reading were spelling and pseudoword reading. Moreover, Swanson et al. found that reading comprehension shared the strongest relationship with word reading, spelling and pseudoword reading. Benson (2008) also found that reading comprehension and reading fluency did not strongly correlate in 
adults. Additionally, consistent with Stanovich's (2005) position, pseudoword reading did not correlate with either verbal or non-verbal IQ. Slower naming speeds on RAN measures were related to difficulty in word reading, spelling and non-word reading (Swanson et al., 2003). In this thesis, RAN Letters but not RAN Objects was related to word reading, spelling and pseudoword reading. Finally, counting span, a measure of working memory, was related to all variables except verbal IQ (Thomson et al., 2006; see also Savage, Lavers, \& Pillay, 2007).

Likewise the correlation between tapping variability (at 1:2 ratio $184 \mathrm{BPM}$ ) and reading skills were similar to those reported by previous research despite the methodological variability in the tapping tasks that have been used (e.g., Chiappe et al., 2002; Ramus et al., 2003; Thomson et al., 2006, Waber et al., 2000; Wolff et al., 1990). The strongest correlations for tapping variability at the 1:2 ratio were with spelling and word reading. This relationship was strongest for the left hand. Similar results have also been reported by Chiappe et al. (2002) who found a strong correlation between tapping variability (i.e., 1:1 alternation) and Word identification $(r=-.41)$ and Spelling $(r=-.45)$. Thompson et al. (2006) also found that greater variability was related to greater difficulty on a word reading test. Moreover, they found that tapping variability was related to working memory and this finding was also supported in this thesis. Finally, increased tapping variability was associated with longer naming times on both RAN Letters and Objects which had also been previously identified by Wolff et al. (1984) and Wolff et al. (1995). This is in contrast to both Thomson et al. and Chiappe et al. who did not report an association between RAN time and tapping variability. However, as previously reported, Thomson et al. used only a single hand tapping paradigm and Chiappe et al. used the 1:1 
tapping in alternation paradigm. Tapping variability was not associated to IQ and again this finding is similar to the one reported by Waber et al. (2000). Tapping variability was also modestly related to passage comprehension and reading fluency. Overall tapping variability was related to multiple aspects of reading, however, the strongest relationship was between word reading and spelling.

To further examine the relationship between tapping variability and reading stepwise regressions were used to determine if tapping variability predicted unique variance in any aspect of reading regardless of phonological processing, IQ, RAN and working memory. The 1:2 ratio task best differentiated among groups and all groups tapped with more variability at $184 \mathrm{BPM}$ at the 1:2 ratio (at least in the left hand). It was hypothesized that tapping variability elicited from the optimal tapping condition (e.g., 1:2 ratio at $184 \mathrm{BPM}$ ) would explain unique variance in decoding, single word reading, spelling, and reading fluency regardless of potential contributing factors such as working memory, IQ, RAN, and phonological processing. Thus, better tappers (i.e., those with low tapping variability) would also be better on tasks of phonological processing, sight word reading, spelling, and reading fluency. Timing stability is hypothesized to be an outward manifestation of temporal processing and may share or overlap with the same cognitive networks as language (Stanford \& Barrett, 1996; Waber et al., 2000; Wolff, 1993). It should be noted, however, that there is no discernible direct or causal association between tapping variability and impaired reading.

Tapping variability of the left-hand at a (1:2) ratio (184 BPM) predicted unique variance in two literacy measures; Word Identification and Spelling. The relationship was such that increased tapping variability in the left hand was related to decreased 
performance on word reading and spelling even with strong predictor variables such as word attack in the model. This is clearly the most important finding of this study.

Tapping variability accounted for approximately 5 percent of the unique variance in word identification even when strong predictors of reading such as phonological processing, verbal IQ, memory and RAN Letters were entered into the model. The results of the regression model for Word identification also confirmed previous research indicating that phonological processing is a key variable that underlies word recognition (Chiappe et al., 2002; Ramus et al., 2003; Vellutino, Fletcher, Snowling, \& Scanlon, 2004). General knowledge of words also contributed uniquely to performance on word identification. This finding also makes intuitive sense in that knowledge of the meaning of words would increase sight vocabulary (Kirby, Desrochers, Roth, \& Lai, 2008). The results may also be specific to the tapping variability produced in a 1:2 ratio tapping condition because previous research has failed to find a unique relationship between tapping variability on 1:1 alternation tasks and word reading (e.g., Chiappe et al., 2002). Ramus et al. (2003) had also rejected the idea that tapping variability (which was part of a composite variable that also included motor skill measures) could explain unique variance in tapping ability. Yet, I have already argued that the speed at which participants were asked to execute the tapping task may not have produced a reliable measure because it was at the limit of the upper temporal threshold (see Repp, 2005). On the other hand, the results of the present study were similar to the results reported by Waber et al. (2000) who found that tapping variability was related to decreased performance on word reading, spelling and mathematics in children. 
Additionally, the present study also found that tapping variability explained approximately 5 percent of the variance associated in spelling. A similar finding was previously reported by Wolff et al. (1996) who found that impaired temporal resolution was related to overall spelling errors and more specifically to the percentage of dysphonetic spelling errors. Wolff et al. (1996) examined dysphonetic spelling errors as a measure of phonological processing. They tentatively theorized that temporal resolution was related to phonological processing because tapping variability accounted for a small amount of variance in dysphonetic spelling errors. However, Wolff et al. cautioned that other experimental measures of phonological processing such as pseudoword reading should be used to verify this prediction. Although the proportion of dysphonetic spelling errors was not examined in the present study, a measure of pseudoword reading was used to determine if temporal resolution was related to phonological processing. The results of the stepwise regression analyses did not support the hypothesis that impaired temporal resolution underlies phonological processing. Therefore, the variance that tapping variability explained in word reading and spelling does not simply underlie impairments in phonological processing. Rather, the results indicate that tapping may be related to a more lexical route to word reading and spelling (Coltheart et al., 1993).

Interestingly, a meta-analysis recently reported that real word reading was best correlated with spelling, and pseudoword reading (Swanson et al., 2006). The results of this thesis also converge on the idea that pseudoword reading is a strong predictor of word identification and spelling. Furthermore, inconsistency in tapping explained additional variance in word identification and spelling that could not be accounted for by 
phonological processing. So what is it about tapping variability that might contribute to both single word reading and spelling but not pseudoword reading?

Variance explained in word reading and spelling was related to the variability in the left hand which is controlled by the right hemisphere of the brain (Banich, 2004; Springer \& Deutsch, 1993). The left hemisphere is known to be specialized for language processing. Thus, it is counterintuitive that tapping in the left hand should predict reading and spelling ability (Springer \& Deutsch, 1993). Before discussing how tapping may be related to language, it must be acknowledged that left handedness can be an indication of atypical lateralization. Nonetheless, analyses that were run both with and without left handed participants yielded the same results. As will be discussed in the next section, the results of rhythmic finger tapping almost certainly do not reflect general deficits in motor movement or simple timing. It is therefore speculated that tapping measures some aspect of temporal processing between the hemispheres.

The production of rhythmic movement is often used to study event timing (Ivry \& Richardson, 2002) and thus rhythmic tapping can be utilized to examine temporal processing. The term temporal refers generally to the way humans process time or generate timed responses (Mauk \& Buonomano, 2004). Temporal processing is the ability to decode the temporal properties of an event and includes "the order, interval, and duration of sensory and motor events" (Mauk \& Buonomano, 2004; p. 308). Moreover, the integrity of the cerebellum is essential in the coordination of timed movement (Ivry et al., 2001; Ivry et al., 2002; Ivry \& Spencer, 2004).

Theoretically, the instability of tapping observed in the dyslexic group may be indicative of a subtle disruption in the cerebellum that, in turn, affects the simple 
precision of timed events (Fawcett \& Nicolson, 1992; see also Ivry et al., 2001). For example, damage to the cerebellum could affect precise timing resulting in increased variability in the output of evenly timed motor responses such as finger tapping. However, Wolff and colleagues suggested that differences in bimanual tapping cannot be fully reduced to a specific deficit in timing (Wolff, 1993; Wolff et al., 1990). If individuals with dyslexia experienced a specific difficulty in timing, differences would also be apparent on simpler rhythmic tapping tasks (i.e., alternation and symmetrical). Group differences in timing stability were only present on the ratio task and this was also found by Wolff et al. (1990). This indicates that individuals with dyslexia were not impaired in tasks that require simple precision of motor movement. Specifically, differences did not appear when the temporal representations of the response goals for each fingers were the same (i.e., alternation or symmetrical).

To this point, it has been argued that differences in tapping variability cannot be reduced to basic deficits in timing because groups did not differ on the easier tapping tasks. In addition, groups did not differ by speed indicating that group differences could not be accounted for by an inability on the part of individuals with dyslexia to process temporal information at a fast rate. Therefore, the essential difference between reading groups was apparent only at the ratio task as previously argued by Wolff et al. (1990).

Consequently, Wolff and colleagues hypothesized that tapping instability may result from impaired interhemispheric communication (Badian \& Wolff, 1977; Wolff 1993; Wolff et al. 1990). Interhemispheric communication, which is primarily facilitated through the corpus callosum, refers to the exchange or coordination of information between the two hemispheres (Banich \& Shenker, 1994). Thus, tapping variability in the 
ratio task could reflect that information transfer is not efficient (i.e., the speed of transfer is too slow); that information transfer is efficient, but that the content is degraded; or that information transfer is affected by a failure to suppress or inhibit redundant information (Wolff, 1993; Wolff et al., 1990). There has been some anatomical evidence to suggest that the corpus callosum in individuals with dyslexia is abnormal and may indeed influence the way information is processed between the hemispheres (see Beaton, 1997; Galaburda, 1993).

In addition, Weems and Zaidel (2004) recently reported evidence of right hemisphere involvement during word recognition using a lexical decision task. In a subsequent study, Weems and Zaidel (2005) found evidence of interhemispheric communication during word recognition tasks. The authors concluded that interhemispheric communication was common during word recognition and the right hemisphere contributes to lexical processing, which leads to significant performance benefits in reading.

Henderson, Barca, and Ellis (2007) also found that adult non-disabled readers were more accurate at word identification when the word was presented to both the right and left visual field (i.e., bilateral advantage). In contrast, the dyslexic participants did not show this bilateral advantage. Interestingly, word identification to the left hemisphere only did not differ between control participants and those with dyslexia. However, word presentation to the right hemisphere resulted in decreased performance in the dyslexic participants in comparison with controls. The authors concluded that dyslexic participants may have difficulty with interhemispheric integration because they did not show the bilateral redundancy gain that control participants exhibit (Henderson et al., 2007). Thus, 
inefficient callosal transfer of written words from the right hemisphere might be the unique variance that tapping explains in reading and spelling

This hypothesis is put forth tentatively, however, as alternate explanations are available. For instance, this study only investigated a 1:2 tapping ratio. It is possible that word reading and spelling were related to the variability in the left finger because the task required the left finger to inhibit a response on every other signal of the metronome. Theoretically, it could be the suppression of information through the corpus callosum that explains variance in reading and spelling. Thus, to rule out this possibility, the tapping ratio should also be investigated with both a $2: 1$ and 1:2 ratio.

\section{Limitations}

An issue raised in the present study is that tapping variability did not appear to be specifically tied to poor word identification and decoding skills. On the other hand, participants in the LD/ADHD group who did not meet the criterion for inclusion in the dyslexia group showed more tapping variability in the 184 BPM 1:2 ratio condition than the control participants. Waber et al. (2000) also found that increased tapping variability also seemed to be a characteristic of children with general learning disabilities that were not specific to reading. However, the results of this study pertaining to the LD/ADHD group should be interpreted with extreme caution because of the small sample size.

A second limitation reflects the difficulty in operationalizing dyslexia. Some researchers categorize participants as dyslexic or non-dyslexic if a participant has a psycho-educational assessment conducted by a registered psychologist that clearly diagnosis dyslexia. However, the majority of studies using this criterion are conducted in Britain (e.g., Ramus et al., 2003; Thomson et al., 2006). In contrast, some studies define 
dyslexia by setting a performance criterion based test of basic reading skills that are conducted at the time of participation (e.g., Chiappe et al., 2002; Shaywitz et al., 2007; Wolff et al. 1990). In Canada, dyslexia is categorized as a type of learning disability that affects reading (LDAO, 2003). As a result, a clear diagnostic statement on a psychoeducational assessment in Canada typically, but not always states a learning disability. Therefore, this study defined dyslexia by selecting participants who scored lower than the $26^{\text {th }}$ percentile on the average of the word identification test and word attack test. This criterion was set based on past research (e.g., Chiappe et al., 2002; Shaywitz et al., 2007) and also on a current working definition of dyslexia that describes reading impairments specifically at the level of single word identification and phonological processing (Mapou, 2008). In at least three cases, this criterion excluded participants who reported having an LD that was classified as dyslexia because their reading scores were found to be above the $26^{\text {the }}$ percentile. Furthermore, a number of participants in the LD/ADHD group reported having difficulty with reading. This finding leaves open the possibility that the DD group and LD/ADHD group were actually not qualitatively different.

The third limitation was the use of the Woodcock Johnson test of achievement. The WJ reading tests are widely used in populations with reading disabilities (e.g., Chiappe et al., 2002; Moore et al., 1995; Shaywitz et al., 2007). The WJ has also been reported to have good psychometric properties and the normative data is based on a large sample (e.g., Strauss et al., 2006). Results from this study suggests that the WJ is a good test for examining below average abilities but not necessarily good for examining above average abilities. In the control group, very few participants achieve standard scores above 115 on word identification, word attack, and passage comprehension. Closer 
examination of the normative data revealed that, in some cases, depending on a participant's age, a near perfect score resulted in a standard score of about 112. Thus, future studies should choose tests that adequately assess a wider range of reading abilities. Similarly, although Elisions is a popular test of phonological ability, it was too easy for control participants who scored near ceiling.

\section{Conclusion}

This thesis first independently replicated the results of Wolff et al. (1990). That is, participants with dyslexia tapped with greater variability at the (1:2) ratio regardless of rate and did not differ from the control participants at the easier (1:1) conditions. This finding did not appear to be specific to dyslexia because the LD/ADHD group also tapped with more variability at the (1:2) ratio. This thesis also showed that tapping variability predicted unique variance in word identification and spelling. However, phonological processing predicted the largest amount of variance in both word identification and spelling. This finding is consistent with the existing literature. Contrary to expectation, tapping variability did not predict variance in phonological processing or reading fluency. Although unpaced tapping requires working memory (Wing, 2002), tapping variability explained unique variance in word identification and spelling even when working memory had been controlled. The brain regions that may be associated with this finding are the cerebellum and the corpus callosum. However, the exact nature of the relationship between tapping variability, word identification, and spelling is speculative at best. 


\section{References}

American Psychiatric Association (2000). Diagnostic and statistical manual of mental disorders (4th ed., revised). Washington, DC, American Psychiatric Association.

Au, A., \& Lovegrove, B. (2001). Temporal processing ability in above average and average readers. Perception \& Psychophysics, 63, 148-155.

Baddeley, A., Gathercole. S., \& Papagno, C. (1998). The phonological loop as a language learning device. Psychological Review, 105, 158-173.

Badian, N. A., \& Wolff, P. (1977). Manual asymmetries of motor sequencing in boys with reading disability. Cortex, 13, 343-349.

Banich, M. T. (2004). Cognitive neuroscience and neuropsychology-2nd edition. New York: Houghton Mifflin Company.

Banich, M. T., \& Shenker, J. I. (1994). Investigations of interhemispheric processing: Methodological considerations. Neuropsychology, 8, 263-277.

Beaton, A. (1997). The relation of the planum temporale asymmetry and morphology of the corpus callosum to handedness, gender, and dyslexia: A review of the evidence. Brain and Language, 60, 255-322.

Benson, N. (2008). Cattell-Horn-Carroll CognitiveAbilities and Reading Achievement. Journal of Psychoeducational Assessment, 26, 27-41.

Bloom, J. S., \& Hynd, G. W. (2005). The role of the corpus callosum in interhemispheric transfer of information: Excitation or inhibition? Neuropsychology Review, 15, 59-71. 
Boder, E. (1973). Developmental dyslexia: A diagnostic approach based on three atypical reading-spelling patterns. Developmental Medicine and Child Neurology, 15, 663687.

Bogen, J. E. (2000). Split-brain basics: Relevance for the concept of one's other mind. Journal of the American Academy of Psychoanalysis, 28, 341-369.

Case, R., Kurland, M. D., \& Goldberg, J. (1982). Operational efficiency and the growth of short-term memory span. Journal of Experimental Child Psychology, 33, 386404.

Chiappe, P., Stringer, R., Siegel, L. S., \& Stanovich, K. E. (2002). Why the timing deficit hypothesis does not explain reading disabilities in adults. Reading and Writing: An Interdisciplinary Journal, 15, 73-107.

Coltheart M., Curtis, B., Atkins, P., \& Haller, M. (1993). Models of reading aloud: Dualroute and parallel-distributed-processing approaches. Psychological Review. 100, $589-608$.

Conway, A. R. A., Kane, M. J., Bunting, M. F., Hambrick, D. Z., Wilhelm, O., \& Engle, R. W. (2005). Working memory span tasks: A methodological review and user's guide. Psychonomic Bulletin and Review, 12, 769-786.

Damasio, A. R., \& Geschwind, N. (1984). The neural basis of language. Annual Review of Neuroscience, 7, 127-147.

Denckla, M. B., \& Rudel, R. (1974). Rapid "automatized" naming of pictured objects, colors, letters and numbers by normal children. Cortex, 10, 186-202.

Engle, R. W. (2007). Counting span (E-Prime) [Computer software]. Retrieved December 18, 2007 from psychology.gatech.edu/renglelab/ 
Farmer, M. E., \& Klein, R. (1995). The evidence for a temporal processing deficit linked to dyslexia: A review. Psychonomic Bulletin \& Review, 2, 460-493.

Fawcett, A. J., \& Nicolson, R. I. (1992). Automatisation deficits in balance for dyslexic children. Perceptual and Motor Skills, 75, 507-529.

Galaburda, A. M. (1993). Neuroanatomic basis of developmental dyslexia. Neurologic Clinics, 11, 161-173.

Geffen, G., Nilsson, J., Quinn, K., \& Teng, E. L. (1985). The effect of lesions of the corpus callosum on finger localization. Neuropsychologia, 23, 497-514.

Gladstone, M., Best, C. T., \& Davidson, R. J. (1989). Anomalous bimanual coordination among dyslexic boys. Developmental Psychology, 25, 236-246.

Habib, M. (2000). The neurological basis of developmental dyslexia and overview and working hypothesis. Brain, 123, 2373-2399.

Hervey, A. S., Epstein, J. N., \& Curry, J. F. (2004). Neuropsychology of adults with attention-deficit/hyperactivity disorder: A meta-analytic review. Neuropsychology, 18, 485-503.

Helland, T., \& Asbjornsen, A. (2004). Digit span in dyslexia: Varieties according to language comprehension and mathematical skills. Journal of Clinical and Experimental at Neuropsychology, 26, 31-42.

Henderson, L., Barca, L., \& Ellis, A. W. (2007). Interhemispheric cooperation and noncooperation during word recognition: Evidence for callosal transfer dysfunction in dyslexic adults. Brain and Language, 103, 276-291.

Ivry, R. B., Diedrichsen, J., Spencer, R. M. C., Hazeltine, E., \& Semjen, A. (2004). A cognitive neuroscience perspective on bimanual coordination. In S. Swinnen and 
J. Duysens (Eds.), Neuro-behavioral Determinants of Interlimb Coordination (pp. 259-295). Boston: Kluwer Academic Publishing.

Ivry, R. B., Justus, T. C., \& Middleton, C. (2001). The cerebellum, timing, and language: Implications for the study of dyslexia. In M. Wolf (Ed.), Dyslexia, Fluency, and the Brain (pp. 189-211).Timonium, MD: York Press.

Ivry, R.B. \& Spencer, R.(2004). The neural representation of time. Current Opinion in Neurobiology, 14, 225-232.

Ivry, R.B., Spencer, R.M., Zelaznik, H.N., \& Diedrichsen, J. (2002). The cerebellum and event timing. In S.M. Highstein and W.T. Thach (Eds.), The Cerebellum: Recent Developments in Cerebellar Research. Annals of the New York Academy of Sciences, Vol. 978. New York: New York Academy of Sciences (pp 302-317).

Kaufman, A. S., \& Kaufman, N. (2004). Kaufman-Brief Intelligence Test Second Edition. Richmond Hill, ON: PSYCAN Corporation.

Kirby, J., Desrochers, A., Roth, L., \& Lai, S. S. (2008) Longitudinal predictors of word reading development. Canadian Psychology/Psychologie canadienne. Special Issue: Literacy development in Canada, 49, 103-110.

Learning Disabilities Association of Canada (2002). Learning disabilities: A new definition. Retrieved August 13, 2008, from http://www.ldao.ca/documents/Definition_and_Suporting

LeMay, M. (1982). Morphological aspects of human brain asymmetry: An evolutionary perspective. Trends in Neurosciences, 5, 273-275.

Lyon, R. G. (1995). Toward a definition of dyslexia. Annals of Dyslexia, 45, 3-27. 
Lyon, R. G. (1996). Learning disabilities. The Future of Children, 6(1), 54-76. Retrieved March 1, 2006, from http://www.futureofchildren.org/usr_doc/vol6no1ART4.pdf

Lyon, R. G., Shaywitz, S. E., \& Shaywitz, B. A. (2003). Part 1: Defining dyslexia, comorbidity, teachers' knowledge of language and reading. Annals of Dyslexia, $53,1-14$.

Mapou, R. (2008, February). Research-based assessment of learning disabilities and attention-deficit hyperactivity/disorder (ADHD) in adults. Paper presented at the annual meeting of the International Neuropsychological Society Continuing Education Program, Hawaii.

Mauk, M. D., \& Buonomano, D. V. (2004). The neural basis of temporal processing. Annual Review of Neuroscience, 27, 307-340.

Miles, T. L., Wheeler, T. J., \& Haslum, M. N. (2003). The existence of dyslexia without severe literacy problems. Annals of Dyslexia, 53, 340-354.

Moore, L. H., Brown, W. S., Markee, T. E., \& Theberge, D. C. (1995). Bimanual coordination in dyslexic adults. Neuropsychologia, 33, 781-793.

Moore, L. H., Brown, W. S., Markee, T. E., Theberge, D. C., \& Zvi, J. C. (1996). Callosal transfer of finger localization information in phonologically dyslexic adults. Cortex, 32, 311-322.

Nicolson, R. I., \& Fawcett, A. J. (1990). Automaticity: A new framework for dyslexia research? Cognition, 35, 159-182.

Nicolson, R. I., Fawcett, A. J., \& Dean, P. (2001). A TINS debate - hindbrain versus the forebrain: A case for cerebellar deficit in developmental dyslexia. Trends in Neuroscience, 24, 508-511. 
Pitre, S. (2006). Hand Tapping Software Version 2.0 User Guide. School of Computer Science, Carleton University.

Ramus, F. (2004). Neurobiology of dyslexia: A reinterpretation of the data. Trends in Neuroscience, 27, 720-726.

Ramus, F., Rosen, S., Dakin, S., Day, B., Castello, J., \& White, S. (2003). Theories of developmental dyslexia: Insights from a multiple case study of dyslexic adults. Brain, 126, 841-865.

Ramus, F., Pidgeon, E., \& Frith, U. (2003). The relationship between motor control and phonology in dyslexic children. Journal of Child Psychology and Psychiatry and Allied Disciplines, 44, 712-722.

Repp, B. H. (2003). Rate limits in sensorimotor synchronization with auditory and visual sequences: The synchronization threshold and the benefits and costs of interval subdivision. Journal of Motor Behavior, 35, 355-370.

Repp, B. H. (2005). Sensorimotor synchronization: A review of the tapping literature. Psychonomic Bulletin \& Review, 12, 969-992.

Repp, B. H. (2006). Rate limits of sensorimotor synchronization. Advances in Cognitive Psychology, 2, 163-181.

Repp, B. H. (2007). Perceiving the numerosity of rapidly occurring auditory events in metrical and non-metrical contexts. Perception \& Psychophysics, 69, 529-543.

Rice, M., \& Brooks, G. (2004, May). Developmental dyslexia in adults: A research review. London: National Research and Development Centre for Adult Numeracy and Literacy. Retrieved September 8, 2006, from http://www.nrdc.ord.uk 
Robichon, F. \& Habib, M. (1998). Abnormal callosal morphology in male adult dyslexics: Relationships to handedness and phonological abilities. Brain and Language, 62, 127-146.

Rousselle, C., \& Wolff, P. H. (1991). The dynamics of bimanual coordination in developmental dyslexia. Neuropsychologia, 29, 907-924.

Savage, R. (2004). Motor skills, automaticity and developmental dyslexia: A review of the research literature. Reading and Writing: An Interdisciplinary Journal, 17, 301-324.

Savage, R., Lavers., N., \& Pillay, V. (2007). Working memory and reading difficulties: What we know and what we don't know about the relationship. Educational Psychology Review, 19, 185-221.

Semjen, A., \& Ivry, R. B. (2001). The coupled oscillator model of between-hand coordination in alternate-hand tapping: A reappraisal. Journal of Experimental Psychology: Human Perception and Performance, 27, 251-265.

Semjen, A., \& Summers, J. J. (2002). Timing goals in bimanual coordination. Quarterly Journal of Experimental Psychology: Section A, 55, 155-171.

Semjen, A., \& Vos, P. G. (2002). The impact of metric structure on performance stability in bimanual 1:3 tapping. Psychological Research, 66, 50-59.

Schneider, W., Eschman, A., \& Zuccolotto, A. (2002a). E-Prime User's Guide. Pittsburgh: Psychology Software Tools Inc.

Schneider, W., Eschman, A., \& Zuccolotto, A. (2002b). E-Prime Reference Guide. Pittsburgh: Psychology Software Tools Inc. 
Shaywitz, B. A., Skudlarski, P., Holahan, J. M., Marchione, K. E., Constable, R. T., Fulbright, R. K., et al. (2007). Age-related changes in reading systems of dyslexic children. American Neurological Association, 61, 363-370.

Smith-Spark, J. H., \& Fisk, J.E. (2007). Working memory functioning in developmental dyslexia. Memory, 15, 34-56.

Snowling, M. J. (1998). Dyslexia as the phonological deficit: Evidence and implications. Child Psychology \& Psychiatric Review, 3, 4-11.

Snowling, M. J. (2001). From language to reading and dyslexia. Dyslexia, 7, 37-46. Snowling, M. J., \& Hayiou-Thomas, M. E. (2006). The dyslexia spectrum continuities between reading, speech, and language impairments. Topics in Language Disorders, 26, 110-126.

Springer, S. P., \& Deutsch, G. (1993). Left Brain, Right Brain (4th ed.). New York: W. H. Freeman and Company.

Standing Committee on a Human Resource Development and the Status of Persons with Disabilities, Parliament of Canada. (2003). Raising Adult Literacy Skills: The Need for a Pan-Canadian Response. Report to the House, June 9, 2003. Minutes of Proceedings and Evidence, meeting number 35. Retrieved August. 31, 2006, from the Government of Canada, House of Commons site: www.parl.gc.ca/InfocomDoc/37/2/HUMA/Studies/Reports/humarp03/humarp03e.pdf

Stanford, M. S. \& Barratt, E. S. (1996). Verbal skills, finger tapping, and cognitive tempo define a second-order factor of temporal information processing. Brain and Cognition, 31, 35-45. 
Stanovich, K. (1993). A model for studies of reading disability. Developmental Review, $13,225-245$.

Stanovich, K. E. (2005). The future of a mistake: Will discrepancy measurement continue to make the learning disabilities field a pseudoscience? Learning Disability Quarterly, 28, 103-106.

Stein, J. (2001). The magnocellular theory of developmental dyslexia. Dyslexia, 7, 7-36.

Stein, J., \& Walsh, V. (1997). To see but not to read; the magnocellular theory of dyslexia. Trends in Neurological Science, 20, 147-152.

Strauss, E., Sherman, E., \& Spreen, O. (2006). A compendium of neuropsychological tests: Administration, and norms, and commentary. New York: Oxford University Press.

Studdert-Kennedy, M., \& Mody, M. (1995). Auditory temporal perception deficits in the reading-impaired: A critical review of the evidence. Psychonomic Bulletin \& Review, 2, 508-514.

Swanson, L. H., Trainin, G., Necoechea, D. M., \& Hammill, D.D. (2003). Rapid naming, phonological awareness, and reading: A meta-analysis of the correlation evidence. Review of Educational Research Winter, 73, 407-440.

Tallal, P. (1984) Temporal or phonetic processing deficit in dyslexia? That is the question. Applied Psycholinguistics, 5, 167-169.

Temple, C. M., Jeeves, M. A., \& Vilarroya, O. O. (1990). Reading in callosal agenesis. Brain and Language, 39, 235-253. 
Thomson, J. M., Fryer, B., Maltby, J., \& Goswami, U. (2006). Auditory and motor rhyming awareness in adults with dyslexia. Journal of Research in Reading, 29, 334-348.

Unsworth, N., Heitz, R. P., Schrock, J. C., \& Engle, R. W. (2005). An automated version of the operation span task. Behavior Research Methods, 37, 498-505.

Vellutino, F. R., Fletcher, J. M., Snowling, M. J., \& Scanlon D. M. (2004). Specific reading disabilities (dyslexia): What have we learned in the past four decades? Journal of Child Psychology and Psychiatry, 45, 2-40.

von Plessan, K., Lundervold, A., Duta, N., Heiervang, E., Klauschen., F., Smievoll, A., et al. (2002). Less developed corpus callosum in dyslexic subjects a structural MRI study. Neuropsychologia, 40, 1035-1044.

Waber, D. P., Weiler, M. D., Bellinger, D. C., Marcus, D. J., Forbes, P. W., Wypij, D., et al. (2000). Diminished motor timing control in children referred for diagnosis of learning problems. Developmental Neuropsychology, 17, 181-197.

Wagner, R., Torgesen, J., \& Rashotte, C. (1999).Comprehensive Test of Phonological Processing (CTOPP). Ontario: PSYCAN Corporation.

Weems, S. A., \& Zaidel, E. (2004). The relationship between reading ability and lateralized lexical decision. Brain and Cognition, 55, 507-515.

Weems, S. A., \& Zaidel, E. (2005). Repetition priming within and between the two cerebral hemispheres. Brain and Language, 93, 298-307.

White, S., Frith, U., Milne, E., Rosen, S., Swettenham, J., \& Ramus, F. (2006). A double dissociation between sensorimotor impairments and reading disability: A 
comparison of autistic and dyslexic children. Cognitive Neuropsychology, 23, 748-761.

White, S., Milne, E., Rosen, S., Hansen, P., Swettenham, J., Frith, U., et al., (2006). The role of sensorimotor impairments in dyslexia: a multiple case study of dyslexic children. Developmental Science, 9, 237-269

Wilkinson, G. S., \& Robertson, G. R. (2006). The Wide Range Achievement Test - 4th edition (WRAT-4). Wilmington, Delaware: Psychological Assessment Resources, Inc.

Wing, A. (2002). Voluntary timing and brain function: An information processing approach. Brain and Cognition, 48, 7-30.

Witelson, S. F. (1995). Neuroanatomical basis of hemispheric functional specialization in the human brain: Possible developmental factors. In F.L Kitterle (Ed.), Hemispheric communication mechanisms and models (pp. 61-78). New Jersey: Lawrence Erlbaum associates, Inc.

Wolf, M., Bowers, P. G., \& Biddle, K. (2000). Naming speed processes, timing, and reading: A conceptual review. Journal of Learning Disabilities, 33, 387-407.

Wolff, P. H. (1993). Impaired temporal Resolution in Developmental Dyslexia. Annals New York Academy of Science, 682, 87-103.

Wolff, P. H. (2002). Timing precision and rhythm in developmental dyslexia. Reading and Writing, 15, 179-206

Wolff, P. H., Cohen, C., \& Drake, C. (1984). Impaired motor-timing control in specific reading retardation. Neuropsychologia, 22, 587-600. 
Wolff, P. H., Melngailis, I., \& Kotwics, K. (1996). Family Patterns of Developmental Dyslexia Part III: Spelling Errors as Behavioral Phenotype. American Journal of Medical Genetics (Neuropsychiatric Genetics), 67, 378-386.

Wolff, P. H., Melngailis, I., Obregon, M., \& Bedrosian, M. (1995). Family patterns of developmental dyslexia, part II: Behavioral phenotypes. American Journal of Medical Genetics (Neuropsychiatric Genetics), 60B, 494-505. (See CORRECTION in, American Journal of Medical Genetics (Neuropsychiatric Genetics), 67, 117.

Wolff, P. H., Michel, G. F., Ovrut, M., \& Drake, C. (1990). Rate and timing precision of motor coordination in developmental dyslexia. Developmental Psychology, 26, 349-359.

Woodcock, R. W., McGrew, K. S., \& Mather, N. (2001). Woodcock-Johnson-III: Tests of Cognitive Abilities. Itasca, IL: Riverside Publishing Co. 


\section{Footnotes}

${ }^{1}$ The tapping data obtained during this initial testing session were lost for 19 participants due to a software error. These 19 participants were contacted and asked to take part in a second testing session to provide new tapping data. Sixteen accepted and received $10 \$$ as compensation. 
Appendix A-Group means and standard errors for tapping variability and tapping

$$
\text { average }
$$

Group means and standard errors for tapping variability and tapping average displayed by Task and by Speed for both the right and left hand.

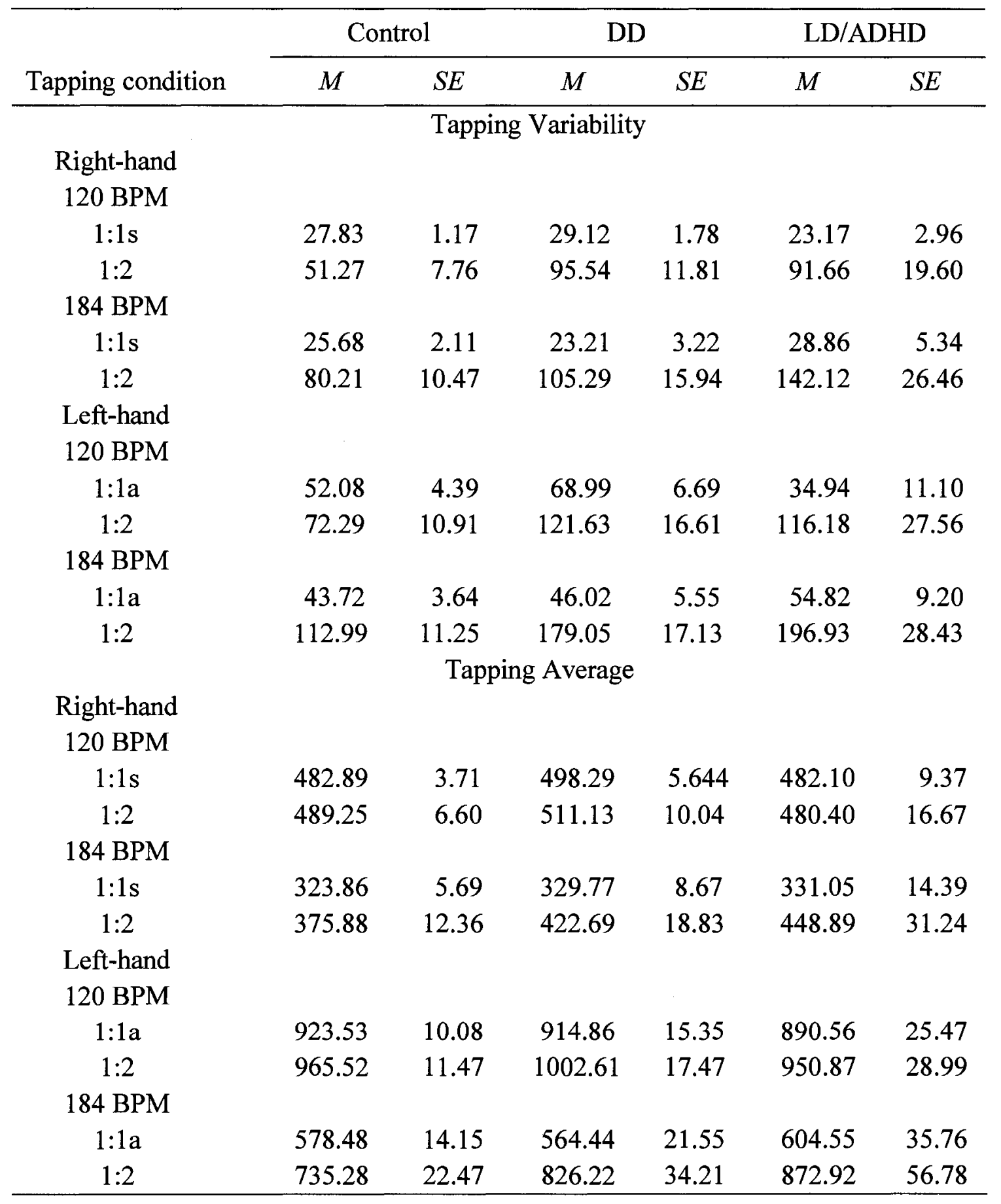

